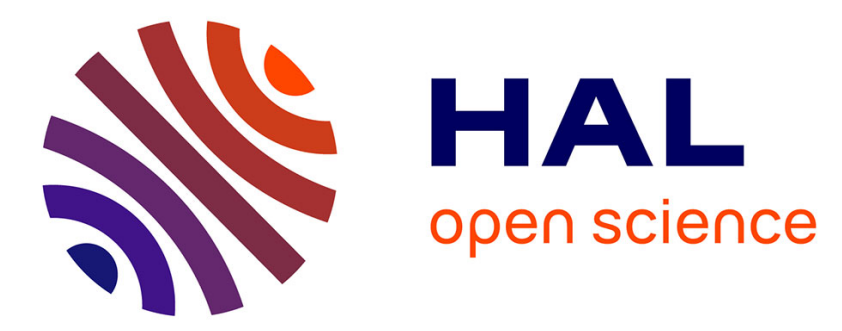

\title{
QoS-Driven Spectrum Sharing for Reconfigurable Intelligent Surfaces (RISs) Aided Vehicular Networks
}

\author{
Yuanbin Chen, Ying Wang, Jiayi Zhang, Marco Di Renzo
}

\section{To cite this version:}

Yuanbin Chen, Ying Wang, Jiayi Zhang, Marco Di Renzo. QoS-Driven Spectrum Sharing for Reconfigurable Intelligent Surfaces (RISs) Aided Vehicular Networks. IEEE Transactions on Wireless Communications, 2021, 20, pp.5969 - 5985. 10.1109/twc.2021.3071332 . hal-03377240

\author{
HAL Id: hal-03377240 \\ https://hal.science/hal-03377240
}

Submitted on 14 Oct 2021

HAL is a multi-disciplinary open access archive for the deposit and dissemination of scientific research documents, whether they are published or not. The documents may come from teaching and research institutions in France or abroad, or from public or private research centers.
L'archive ouverte pluridisciplinaire HAL, est destinée au dépôt et à la diffusion de documents scientifiques de niveau recherche, publiés ou non, émanant des établissements d'enseignement et de recherche français ou étrangers, des laboratoires publics ou privés. 


\title{
QoS-Driven Spectrum Sharing for Reconfigurable Intelligent Surfaces (RISs) Aided Vehicular Networks
}

\author{
Yuanbin Chen ${ }^{\circledR}$, Ying Wang ${ }^{\circledR}$, Member, IEEE, Jiayi Zhang ${ }^{\circledR}$, Senior Member, IEEE, \\ and Marco Di Renzo ${ }^{\circledR}$, Fellow, IEEE
}

\begin{abstract}
Reconfigurable intelligent surfaces (RISs) have the capability of reconfiguring the wireless environment in a favorable way to improve the quality-of-service $(\mathrm{QoS})$ of wireless communications. This makes RISs a promising candidate to enhance vehicle-to-everything (V2X) applications. This paper investigates the spectrum sharing problem in RIS-aided vehicular networks, in which multiple vehicle-to-vehicle (V2V) links reuse the spectrum already occupied by vehicle-to-infrastructure (V2I) links. To overcome the difficulty of acquiring instantaneous channel state information (CSI) due to the fast varying nature of some V2X channels, we rely upon large-scale (slowly varying) CSI in order to fulfill the QoS requirements of V2I and V2V communications. Particularly, we aim to maximize the sum capacity of V2I links that are used for high-rate content delivery and to guarantee the reliability of $\mathrm{V} 2 \mathrm{~V}$ links that are used for the exchange of safety information. The transmit power of vehicles, the multi-user detection (MUD) matrix, the spectrum reuse of $\mathrm{V} 2 \mathrm{~V}$ links, and the RIS reflection coefficients are jointly optimized, which results in a mixed-integer and non-convex optimization problem. To tackle this problem, the outage probability of each $\mathrm{V} 2 \mathrm{~V}$ link is first approximated by introducing an analytical expression to simplify the formulated problem. By leveraging the block coordinate descent (BCD) method, the considered optimization problem is decomposed into three sub-problems, whose optimal solutions are provided independently and updated alternately to obtain a near-optimal solution. Simulation results verify the theoretical analysis and the effectiveness of the proposed algorithm, as well as unveil the benefits of introducing RISs for enhancing the QoS performance of vehicular communications.
\end{abstract}

Index Terms-Vehicular networks, V2X, reconfigurable intelligent surfaces, QoS, resource allocation.

Manuscript received August 25, 2020; revised January 5, 2021 and March 4, 2021; accepted March 29, 2021. Date of publication April 13, 2021; date of current version September 10, 2021. This work was supported by the National Key Research and Development Program of China under Grant 2018YFE0205502. The associate editor coordinating the review of this article and approving it for publication was K. Huang. (Corresponding authors: Ying Wang; Jiayi Zhang.)

Yuanbin Chen and Ying Wang are with the State Key Laboratory of Networking and Switching Technology, Beijing University of Posts and Telecommunications, Beijing 100876, China (e-mail: chen_yuanbin@163.com; wangying@ bupt.edu.cn).

Jiayi Zhang is with the School of Electronic and Information Engineering, Beijing Jiaotong University, Beijing 100044, China (e-mail: jiayizhang@bjtu.edu.cn).

Marco Di Renzo is with Université Paris-Saclay, CNRS, CentraleSupélec, Laboratoire des Signaux et Systèmes, 91192 Gif-sur-Yvette, France (e-mail: marco.di-renzo@universite-paris-saclay.fr).

$\sim$

\section{INTRODUCTION}

$\mathbf{P}$ ROPELLED by the booming development of wireless communication technology, vehicular communications, also known as vehicle-to-everything (V2X) communications, are promising paradigm to support cutting-edge applications, such as vehicular infotainment services and autonomous driving. Recently, the 3rd Generation Partnership Project (3GPP) has been committed to standardizing V2X services in long-term evolution (LTE) and fifth-generation (5G) cellular networks [1]-[3]. In Release 16 [3], a wide variety of new use cases and requirements have been proposed and analyzed to enhance 5G V2X. For example, vehicular entertainment services need high-rate connections with the base station (BS), such as high-data-rate entertainment and dynamic digital map updates. In addition, autonomous driving and vehicle platooning need to exchange safety messages (such as vehicle position, speed, direction, etc.) among adjacent vehicles through vehicle-to-vehicle $(\mathrm{V} 2 \mathrm{~V})$ communications. This, in addition, needs to be achieved with high reliability in order to improve the "cooperation awareness" of all vehicles in the local driving environment. Therefore, it is very important to allocate the communication resources according to the channel conditions and quality of service (QoS) requirements of the vehicle user equipments (VUEs). However, current standards, e.g., IEEE 802.11p and cellular-V2X (C-V2X), do not support the QoS required by advanced vehicular applications, and cannot provide consistent high-data-rate transmission [4]. Therefore, it is indispensable to propose emerging technologies that enable QoS-aware resource allocation solutions in order to support ubiquitous coverage and QoS guarantee in the context of V2X communications.

In order to satisfy and enhance the QoS of V2X communications, reconfigurable intelligent surfaces (RISs) have been spotlighted as an emerging technology for achieving a high spectral and energy efficiency [5]-[7]. RISs are passive array structures that are capable of tuning the phase of each reflecting element on a surface either almost continuously [8] or discretely [9], and at a low power consumption. In particular, RISs are designed to reflect the signal from the BS to a specific receiver so as to strengthen the received signal power, or to suppress the interference for ensuring security or privacy [10]. RISs can be deployed easily on existing infrastructure (such as the facade of buildings), thus reducing the operators' expenses 
and installation complexity [11]. Compared to traditional active antenna arrays equipped with energy-inefficient radio frequency (RF) chains and power amplifiers, RISs with passive reflective elements are cost-effective and energy-efficient. Therefore, these characteristics support the development and deployment of RISs as a promising enabler for optimizing wireless communication systems.

Owing to these considerable advantages, extensive research on RISs has been conducted for application to wireless communications, such as RIS-assisted unmanned aerial vehicle (UAV) systems [12], RIS-assisted multi-group multicast systems [13], and RIS-aided multicell multiuser multiple-input multiple-output (MIMO) systems [14]. In addition, resource scheduling to improve the QoS in RIS-aided wireless communication systems has been investigated by many researchers. In [15], the computation and communication tasks were jointly designed to effectively minimize the computation latency in RIS-aided mobile edge computing (MEC) networks. In [16], by exploiting RISs for downlink multi-user communication from a multi-antenna BS, the system energy efficiency was improved. The joint optimization of the RIS reflection coefficients together with the time-frequency resource block and power allocations was studied in [17]. In [18], the RIS phase shift matrix and power allocation were jointly optimized to maximize the security rate for application to RIS-aided multiple-input single-output (MISO) systems. Under QoS constraints, the authors of [19] studied RIS-aided simultaneous wireless information and power transfer (SWIPT) systems.

Despite the large number of research contributions on RIS-aided wireless networks, research on RIS-aided vehicular networks is still at its infancy. The available research works on RISs-aided vehicular networks are focused on physical layer security [20] and on the outage probability of V2I links [21]. Based on the current state of research, we evince that the potential gains of RIS-aided communications have not been leveraged yet for enhancing V2X communications with QoS-guarantee, especially in harsh transmission environments. Filling this gap is the main objective of the present paper.

This work, in particular, is based on Mode 4 defined in the 3GPP cellular V2X architecture [1]. Within this framework, the vehicles have a pool of radio spectrum resources that can be independently selected for V2V communications [22], [23]. To make full use of the available radio spectrum resources, current recommendations foresee that V2I communications are assigned the radio spectrum in an orthogonal manner, while V2V communications reuse the radio spectrum of V2I communications. Therefore, resource optimization is essential for the design of $\mathrm{V} 2 \mathrm{~V}$ connections in order to devise appropriate spectrum sharing strategies that fulfill different QoS requirements. Such a design is necessary for ensuring the coexistence of V2I and V2V links on a limited frequency spectrum, which, however, leads to more sophisticated interference management schemes. These issues can be tackled by appropriately deploying and optimizing RISs in vehicular networks, since the transmitted signal can be strengthened by appropriately tuning the phase shifts of the reflecting elements of RISs. The signals reflected from the RIS can be added constructively at the desired VUE in order to enhance the received signal power, while they can be superposed destructively at the unintended receiver in order to suppress interference. The present work, in particular, differentiates itself from our previous work [24] in two main aspects. First, an uplink single-input multi-output (SIMO) scenario for the V2I links is considered. Second, we consider the outage probability, i.e., the probability that the instantaneous transmission rate of RIS-aided V2V links is less than a given threshold, for modeling the reliability of V2V links.

Against this background, the main contributions of the present work can be summarized as follows:

- The considered spectrum sharing problem for RIS-aided vehicular networks is based on large-scale (slowly varying) channel state information (CSI) instead of instantaneous CSI, in order to overcome the difficulty of estimating the channel side information in vehicular scenarios with high mobility. To fulfill the QoS requirements in V2X communications, in particular, the transmit power of the VUEs, the multi-user detection (MUD) matrix, the spectrum sharing strategy for the V2V links, and the RIS reflection coefficients are jointly optimized to maximize the sum V2I capacity while guaranteeing the reliability of $\mathrm{V} 2 \mathrm{~V}$ communications. The coupled variables and intricate constraints exacerbate the difficulty of solving the resulting mixed-integer non-convex problem.

- To account for the reliability requirements of $\mathrm{V} 2 \mathrm{~V}$ communications, the outage probability is typically employed as a performance metric. The resulting analytical formulation with a probabilistic constraint is normally unsuitable for optimization and resource allocation. To circumvent this issue, we propose a simple but accurate approximation.

- By leveraging the block coordinate descent (BCD) method, the formulated optimization problem is decomposed into three sub-problems and each of them is tackled independently. First, the joint optimization of the transmit power and the spectrum sharing strategy for the V2V links is considered. Then, the MUD matrix is optimized by invoking the semi-definite relaxation (SDR) method. Finally, a penalty convex-concave procedure $(\mathrm{CCP})$ method is exploited to design the phase shifts of the RIS elements. Furthermore, the solutions of the three subproblems are glued together by utilizing our proposed alternating optimization-based iterative algorithm (AOIA).

- Simulation results are provided to demonstrate the effectiveness of the proposed algorithm and its superiority as compared with several benchmark schemes. In particular, the obtained simulation results show that, by using the proposed resource allocation algorithm, RIS-aided vehicular networks are capable of satisfying different QoS requirements for the V2X links while increasing, as compared with baseline schemes not using RISs, the sum capacity of V2I links. In addition, the obtained numerical results show that the deployment of RISs in vehicular networks can compensate for the channel losses due to the mobility of vehicles, and that large performance gains 
can be obtained provided that the location of the RIS is carefully selected.

The remainder of this paper is organized as follows. Section II introduces the system model and the problem formulation. The outage probability of the $\mathrm{V} 2 \mathrm{~V}$ links is analyzed in Section III. In Section IV, an effective algorithm is proposed, and its complexity and convergence are analyzed. Simulation results are provided in Section V, and, finally, Section VI concludes the paper.

Notations: The following notations and symbols are used throughout this paper. Italic letters denote scalars. Boldface lower- and upper-case letters denote vectors and matrices, respectively. $\mathbb{C}^{M \times N}$ represents the complex space with $M \times N$ dimension, and $\mathbf{I}_{N}$ denotes an $N \times N$ identity matrix. $\{\cdot\}^{*},\{\cdot\}^{T}$, and $\{\cdot\}^{H}$ stand for the conjugate, transpose, and Hermitian (conjugate transpose) operators, respectively. The symbols $|\cdot|$ and $\|\cdot\|$ denote the absolute value of a scalar and the $\ell_{2}$-norm of a vector, respectively. $\odot$ denotes the Hadamard product. $\operatorname{Tr}(\cdot), \operatorname{diag}(\cdot)$ and $\operatorname{rank}(\cdot)$ represent trace, diagonalization and rank of a square matrix, respectively. $\jmath$ is the imaginary unit, i.e., $\jmath^{2}=-1 . \Re\{\cdot\}$ and $\Im\{\cdot\}$ denote the real part and the imaginary part of a complex value, respectively. The distribution of a circularly symmetric complex Gaussian (CSCG) with zero mean and variance $\Sigma$ is denoted by $\mathcal{C N}(0, \Sigma) . \mathbb{E}\{\cdot\}$ denotes the statistical expectation operator. $\chi^{2}(v)$ denotes a chi-square random variable with $v$ degrees of freedom.

\section{SySTEM MODEL}

\section{A. Scenario}

We consider an RIS-aided vehicular communication network with V2I and V2V communication links that support advanced driving services, such as mobile high-data-rate entertainment, autonomous driving, and vehicle platooning, as discussed in 3GPP R16 for cellular V2X communications [3]. As for the V2I mode, we consider an uplink SIMO scenario, in which the vehicles are equipped with a single antenna, and the BS is equipped with a $K$-element uniform linear array (ULA) ${ }^{1}$. The considered vehicular network consists of $M$ cellular-vehicle user equipments (C-VUEs) that communicate with the BS through V2I links, and $L$ pairs of device-to-device vehicle user equipments (D-VUEs) that communicate with each other directly through device-to-device (D2D) communication links. The set of C-VUEs and D2D-V2V pairs is denoted by $\mathcal{M}=\{1,2, \ldots, M\}$ and $\mathcal{L}=\{1,2, \ldots, L\}$, respectively. Due to the spectrum sharing among multiple VUEs, the QoS of the considered vehicular network is impaired by the interference leakage from different types of V2X links. To effectively suppress the interference and guarantee the QoS of each V2X link, an RIS is deployed to enhance the coexistence and performance of multiple V2X links. The RIS is a uniform rectangular array (URA), which contains $N_{\mathrm{h}}$ horizontally arranged and $N_{\mathrm{v}}$ vertically arranged passive reflecting elements. Thus the total number of RIS reflecting elements is $N=N_{\mathrm{h}} \times N_{\mathrm{v}}$.

\footnotetext{
${ }^{1}$ Usually, the SIMO/MISO setup is considered to simplify the analysis [25]. It is worth noting that our work can be extended to MIMO systems.
}

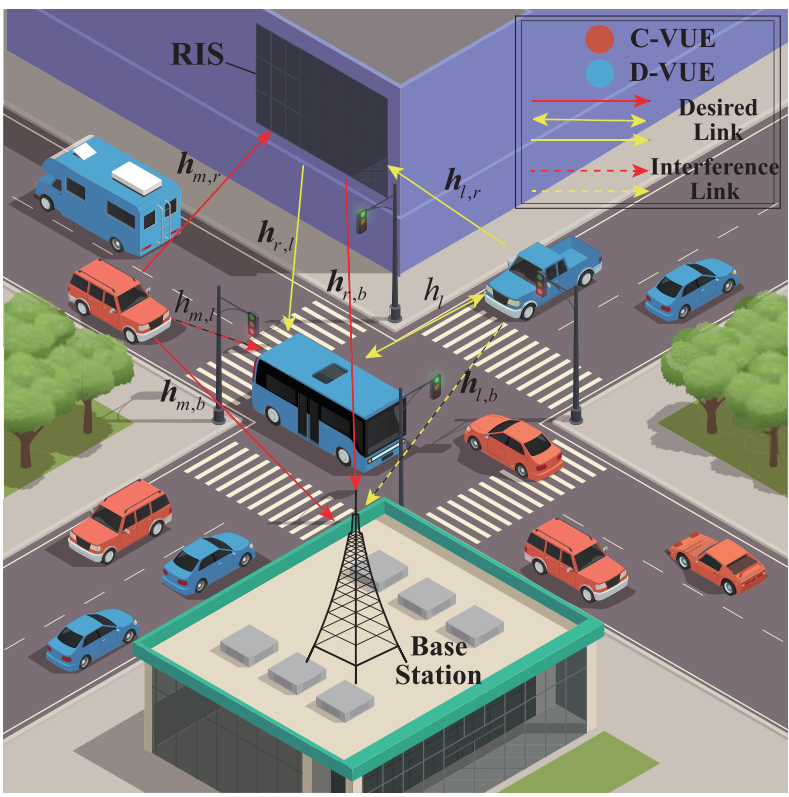

Fig. 1. Reconfigurable intelligent surface aided vehicular networks.

\section{B. Channel Model}

The channel gain between the $m$ th $\mathrm{C}$-VUE and the BS is denoted by $\mathbf{h}_{m, b}=\sqrt{\rho d_{m, b}^{-\alpha_{m, b}}} \tilde{\mathbf{h}}_{m, b} \in \mathbb{C}^{K \times 1}$, where $\rho$ is the path loss at the reference distance $d_{0}=1 \mathrm{~m}, d_{m, b}$ is the distance between the $m$ th C-VUE and the BS, and $\alpha_{m, b}$ is the corresponding path loss exponent. Each element of $\tilde{\mathbf{h}}_{m, b}$ follows an independent and identically distributed (i.i.d.) complex Gaussian distribution with zero mean and unit variance. The channel gain from the RIS to the BS is denoted by $\mathbf{h}_{r, b} \in \mathbb{C}^{K \times N}$, and it follows a Rician distribution

$\mathbf{h}_{r, b}=\sqrt{\rho d_{r, b}^{-\alpha_{r, b}}}\left(\sqrt{\frac{\kappa_{r, b}}{1+\kappa_{r, b}}} \mathbf{h}_{r, b}^{\mathrm{LoS}}+\sqrt{\frac{1}{1+\kappa_{r, b}}} \mathbf{h}_{r, b}^{\mathrm{NLoS}}\right)$,

where $\alpha_{r, b}$ is the path loss exponent of the RIS-BS link, $d_{r, b}$ is the distance from the RIS to the BS, and $\kappa_{r, b}$ is the Rician factor related to small-scale fading. The line-of-sight (LoS) component $\mathbf{h}_{r, b}^{\mathrm{LoS}} \in \mathbb{C}^{K \times N}$ consists of the ULA array response, and each element of the non-LoS (NLoS) component $\mathbf{h}_{r, b}^{\mathrm{NLoS}}$ follows an i.i.d. complex Gaussian distribution with zero mean and unit variance. Since the BS and the RIS are deployed in fixed positions, the RIS-BS link remains static over a long period of time. The channel gain from the $m$ th C-VUE to the RIS is denoted by $\mathbf{h}_{m, r} \in \mathbb{C}^{N \times 1}$, and it follows a Rician distribution similar to (1). As far as the LoS component of the VUE-RIS link $\mathbf{h}_{m, r}^{\mathrm{LoS}}$ is concerned, the angle of arrival (AoA) at the RIS mainly depends on the location of the vehicle, i.e., $\vartheta^{\text {AoA }}=\arccos \left(\left|\mathfrak{x}_{m}-\mathfrak{x}_{r}\right| / d_{m, r}\right)$ [12], where $\mathfrak{x}_{m}, \mathfrak{x}_{r}$ and $d_{m, r}$ denote the locations of the $m$ th C-VUE and the RIS, and the distance from the $m$ th C-VUE to the RIS, respectively.

Along the same lines, we can formulate the desired channel gain of the $l$ th $\mathrm{D} 2 \mathrm{D}-\mathrm{V} 2 \mathrm{~V}$ pair (including the path loss and Rayleigh fading), the channel gain from the transmitter of the $l$ th D2D-V2V pair to the RIS, and the channel gain from 
the RIS to the receiver of the $l$ th $\mathrm{D} 2 \mathrm{D}-\mathrm{V} 2 \mathrm{~V}$ pair, which are denoted by $h_{l}, \mathbf{h}_{l, r} \in \mathbb{C}^{N \times 1}, \mathbf{h}_{r, l} \in \mathbb{C}^{N \times 1}$, respectively. Moreover, let $h_{m, l}$ denote the interference channel gain from the $m$ th C-VUE to the receiver of the $l$ th D2D-V2V pair and let $\mathbf{h}_{l, b} \in \mathbb{C}^{K \times 1}$ denote the interference channel gain from the transmitter of the lth D2D-V2V pair to the BS. The LoS components of $\mathbf{h}_{l, r}, \mathbf{h}_{r, l}$ and $\mathbf{h}_{l, b}$ depend on the AoAs at the RIS and the BS, as well as on the angles of depature (AoDs) at the RIS, which can be calculated according to the vehicles' location information.

Due to the high mobility in V2X communications, it is fairly difficult to obtain accurate estimates of the instantaneous CSI, and may entail a large estimation overhead. In the considered model, the resource allocation and beamforming design are based on a time interval (which is referred to as "block" in the rest of this paper), of the order of hundreds of milliseconds, which consists of several consecutive slots whose duration is of the order of hundreds of microseconds. Since the large-scale fading and the deterministic LoS components of the individual channels are typically determined by the locations of vehicles, which are less likely to change significantly during one block, they are assumed invariant and fixed during one block but are subject to changing from one block to another [23], [26]. As far as the NLoS component of the channel (i.e., small-scale fading) is concerned, we assume that it is constant during one slot but varies independently from one slot to another. Furthermore, we assume that the BS has access only to the statistics of the small-scale fading, and that it does not have access to individual channel realizations [27]. Also, we assume that the CSI is fed back to the BS once per block, which implies that the BS knows only the slowly varying CSI. This requires reduced signaling overhead for reporting the CSI. Based on these assumptions, this paper focuses on system optimization and resource allocation in a particular block. In addition, we assume that the per-block CSI is perfectly available at the BS. Under these assumptions, our analysis provides information on the best achievable performance bounds. The analysis of scenarios with imperfect per-block CSI is postponed to future research.

\section{Communication Protocol}

As for the RIS, we set the amplitude reflection coefficient to one for all reflecting elements and denote the phase reflection coefficient of the $n$th reflecting element by $\theta_{n}=e^{\jmath \varphi_{n}}$, where $\varphi_{n} \in[0,2 \pi)^{2}$. Then, the RIS reflection-coefficient matrix is defined as $\boldsymbol{\Theta}=\operatorname{diag}\left(\left[\theta_{1}, \ldots, \theta_{N}\right]^{T}\right)$. Let the MUD matrix be denoted by $\mathbf{F}=\left[\mathbf{f}_{1}, \ldots, \mathbf{f}_{M}\right] \in \mathbb{C}^{K \times M}$. The received signal at the BS from the $m$ th C-VUE can be formulated as

$$
\begin{aligned}
y_{m}=\mathbf{f}_{m}^{H} & {\left[\sqrt{P_{m}^{c}}\left(\mathbf{h}_{r, b} \boldsymbol{\Theta} \mathbf{h}_{m, r}+\mathbf{h}_{m, b}\right) s_{m}\right.} \\
& \left.+\sum_{l \in \mathcal{L}} x_{m, l} \sqrt{P_{l}^{d}}\left(\mathbf{h}_{r, b} \boldsymbol{\Theta} \mathbf{h}_{l, r}+\mathbf{h}_{l, b}\right) s_{l}+\boldsymbol{n}\right],
\end{aligned}
$$

\footnotetext{
${ }^{2}$ In practice, the phase shifts are selected from a finite number of discrete values that vary between 0 and $2 \pi$ due to hardware limitations [9]. The proposed algorithm provides, therefore, the best-case bound for realistic scenarios.
}

where $s_{m}, s_{l}, P_{m}^{c}$ and $P_{l}^{d}$ represent the transmitted information symbol and the transmit power of the $m$ th C-VUE and of the transmitter of the $l$ th $\mathrm{D} 2 \mathrm{D}-\mathrm{V} 2 \mathrm{~V}$ pair, respectively; and $\mathbf{f}_{m}$ is the $k$ th column of the matrix $\mathbf{F}$. The noise vector is denoted by $\boldsymbol{n}=\left[n_{1}, \ldots, n_{M}\right]^{T}$, and it is assumed that $n_{m} \sim \mathcal{N}\left(0, \sigma^{2}\right), \forall m \in \mathcal{M}$. The binary variable $x_{m, l}$ is employed to indicate whether the $l$ th D2D-V2V pair reuses the frequency spectrum of the $m$ th C-VUE. If spectrum sharing is performed, $x_{m, l}=1$. Otherwise, $x_{m, l}=0$. Similar to [22], [26], we assume that the transmission over the V2I links occurs over orthogonal frequency bands, so that the interference among the V2I links can be ignored. On the other hand, the V2V links reuse the frequency bands of the V2I links, and, therefore, this interference is duly taken into account in our system model. As for the receiver of the $l$ th $\mathrm{D} 2 \mathrm{D}-\mathrm{V} 2 \mathrm{~V}$ pair, the received signal is given by ${ }^{3}$

$$
\begin{aligned}
y_{l}= & \sqrt{P_{l}^{d}}\left(\mathbf{h}_{r, l}^{H} \boldsymbol{\Theta} \mathbf{h}_{l, r}+h_{l}\right) s_{l} \\
& +\sum_{m \in \mathcal{M}} x_{m, l} \sqrt{P_{m}^{c}}\left(\mathbf{h}_{r, l}^{H} \boldsymbol{\Theta} \mathbf{h}_{m, r}+h_{m, l}\right) s_{m}+n_{l},
\end{aligned}
$$

where $n_{l} \sim \mathcal{N}(0,1), \forall l \in \mathcal{L}$. Then, the uplink signal-tointerference-plus-noise ratio (SINR) of the $m$ th C-VUE is given by

$$
\operatorname{SINR}_{m}=\frac{P_{m}^{c}\left|\mathbf{f}_{m}^{H}\left(\mathbf{h}_{r, b} \Theta \mathbf{h}_{m, r}+\mathbf{h}_{m, b}\right)\right|^{2}}{\sum_{l \in \mathcal{L}} x_{m, l} P_{l}^{d}\left|\mathbf{f}_{m}^{H}\left(\mathbf{h}_{r, b} \Theta \mathbf{h}_{l, r}+\mathbf{h}_{l, b}\right)\right|^{2}+\sigma^{2}\left|\mathbf{f}_{m}^{H}\right|^{2}}
$$

and the received SINR of the $l$ th $\mathrm{D} 2 \mathrm{D}-\mathrm{V} 2 \mathrm{~V}$ pair is given by

$$
\operatorname{SINR}_{l}=\frac{P_{l}^{d}\left|\mathbf{h}_{r, l}^{H} \Theta \mathbf{h}_{l, r}+h_{l}\right|^{2}}{\sum_{m \in \mathcal{M}} x_{m, l} P_{m}^{c}\left|\mathbf{h}_{r, l}^{H} \Theta \mathbf{h}_{m, r}+h_{m, l}\right|^{2}+\sigma^{2}} .
$$

Accordingly, the achievable uplink rate of the $m$ th C-VUE and the achievable transmission rate of the $l$ th $\mathrm{D} 2 \mathrm{D}-\mathrm{V} 2 \mathrm{~V}$ pair can be formulated as $R_{m}=\log _{2}\left(1+\operatorname{SINR}_{m}\right)$ and $R_{l}=$ $\log _{2}\left(1+\mathrm{SINR}_{l}\right)$, respectively.

\section{Problem Formulation}

As described in previous text, the V2I links are designed to support mobile high-data-rate entertainment services. Therefore, we are interested in maximizing the sum V2I capacity, i.e., $\sum_{m \in \mathcal{M}} R_{m}$. On the other hand, the $\mathrm{V} 2 \mathrm{~V}$ links are mainly employed for the reliable dissemination of security-critical messages. As far as the V2V are concerned, therefore, the link reliability is the main performance criterion of interest. We use the outage probability to ensure the reliability of $\mathrm{V} 2 \mathrm{~V}$ transmission, which is defined as

$$
\operatorname{Pr}\left(R_{l} \leq R^{\text {th }}\right) \leq P_{\max }^{\text {outage }},
$$

\footnotetext{
${ }^{3}$ The interference caused by spectrum sharing in (2) and (3) cannot be eliminated by the RIS although the reflection coefficients are appropriately configured. The reflected signals can be destructively superimposed at the non-intended receivers to mitigate the interference via appropriately optimizing the RIS.
} 
where $R^{\text {th }}$ is the minimum reliable transmission rate required for each $\mathrm{V} 2 \mathrm{~V}$ link, and $P_{\max }^{\text {outage }}$ is the maximum tolerable outage probability.

On this basis, the spectrum sharing problem studied in this paper encompasses the joint optimization of the transmit power $\mathbf{P}=\left\{P_{m}^{c}, P_{l}^{d}, \forall m, l\right\}$, the MUD matrix $\mathbf{F}$, the spectrum sharing strategy for the V2V links $\mathbf{X}=\left\{x_{m, l}, \forall m, l\right\}$, and the RIS reflection-coefficient matrix $\Theta$. Thus, the optimization problem that ensures the desired QoS requirements for RIS-aided V2X communications, can be formulated as follows

$$
\begin{aligned}
\max _{\{\mathbf{F}, \mathbf{\Theta}, \mathbf{P}, \mathbf{X}\}} & \sum_{m \in \mathcal{M}} R_{m} \\
\text { s.t. } & \operatorname{Pr}\left(R_{l} \leq R^{\mathrm{th}}\right) \leq P_{\max }^{\text {outage }}, \quad \forall l, \\
& \left|\theta_{n}\right|=1, \quad \forall n, \\
& 0 \leq P_{m}^{c} \leq P_{\max }^{c}, \quad \forall m \\
& 0 \leq P_{l}^{d} \leq P_{\max }^{d}, \quad \forall l, \\
& \sum_{l \in \mathcal{L}} x_{m, l} \leq 1, \quad \forall m, \\
& \sum_{m \in \mathcal{M}} x_{m, l} \leq 1, \quad \forall l, \\
& x_{m, l} \in\{0,1\}, \quad \forall m, l,
\end{aligned}
$$

where $P_{\max }^{c}$ and $P_{\max }^{d}$ is the maximum allowed transmit power of each C-VUE and of each transmitter of a D2D-V2V pair, respectively. To elaborate further, (7c) characterizes the unit modulus constraint of the phase shift of each reflecting element, and (7e) characterizes the spectrum sharing protocol for the V2X links, i.e., the spectrum of one C-VUE can be shared with at most one D2D-V2V pair and, conversely, one $\mathrm{D} 2 \mathrm{D}-\mathrm{V} 2 \mathrm{~V}$ pair can only reuse the spectrum of at most one C-VUE. It is worth noting that (7e) reduces the complexity of interference management and serves as a reasonable starting point for resource sharing in vehicular networks [23].

The optimization problem formulated in (7) accounts for different QoS requirements in V2X communications. The main challenge for solving problem (7) lies in three issues: (i) there is no closed-form expression for the constraint in (7b) that fulfills the outage probability constraint for V2V links; (ii) the optimization variables are highly coupled with each other; (iii) the binary variables $\mathbf{X}$ related to spectrum sharing and the unit-modulus constraint in $(7 \mathrm{c})$ render the problem even more difficult to tackle. In order to overcome these challenges, we first compute an analytical expression of the outage probability that appears in ( $7 \mathrm{~b})$, and, then, we propose an effective iterative algorithm for solving (7).

\section{OUtAGE ANALYSis FOR V2V Links}

The outage probability constraint in (7b) can be rewritten as $\operatorname{Pr}\left\{\log _{2}\left(1+\mathrm{SINR}_{l}\right) \leq R^{\text {th }}\right\}=$ $\mathbb{E}\left\{u\left(R^{\text {th }}-\log _{2}\left(1+\operatorname{SINR}_{l}\right)\right)\right\}$, where $u(\cdot)$ is the step function [28]. There exist many smooth approximations for the step function. Let $\hat{u}_{\delta}(\cdot)$ denote a smooth approximation of the step function $u(\cdot)$ with a non-negative smooth parameter $\delta$. One possible and general form of a smooth approximation function is $\hat{u}_{\delta}(z)=\left(1+e^{-\delta z}\right)^{-1}$ [28], where the smooth parameter $\delta$ can be used to control the approximation error. Hence, (7b) can be approximated as

$$
\mathbb{E}\left\{\hat{u}_{\delta}\left(R^{\text {th }}-\log _{2}\left(1+\operatorname{SINR}_{l}\right)\right)\right\} \leq P_{\text {max }}^{\text {outage }}, \forall l .
$$

The difficulty of computing (8) mainly lies in the expectation operator, and a closed-form expression of (8) is essential in order to further analyze the outage probability of V2V links.

Due to the concavity of the step function and by using Jensen's inequality, the left hand side (LHS) of (8) can be upper bounded by

$$
\begin{aligned}
\mathbb{E}\left\{\hat{u}_{\delta}(\right. & \left.\left.R^{\text {th }}-\log _{2}\left(1+\operatorname{SINR}_{l}\right)\right)\right\} \\
& \leq \hat{u}_{\delta}\left(\mathbb{E}\left\{R^{\text {th }}-\log _{2}\left(1+\operatorname{SINR}_{l}\right)\right\}\right) \\
& =\hat{u}_{\delta}\left(R^{\text {th }}-\mathbb{E}\left\{\log _{2}\left(1+\operatorname{SINR}_{l}\right)\right\}\right) .
\end{aligned}
$$

As for $\mathbb{E}\left\{\log _{2}\left(1+\mathrm{SINR}_{l}\right)\right\}$, according to the concavity of the log function and by using Jensen's inequality, we obtain

$$
\begin{aligned}
& \mathbb{E}\left\{\log _{2}\left(1+\mathrm{SINR}_{l}\right)\right\} \\
& \leq \log _{2}\left(1+\mathbb{E}\left\{\mathrm{SINR}_{l}\right\}\right) \\
& \stackrel{(a)}{\approx} \log _{2}\left(1+\frac{\mathbb{E}\left\{P_{l}^{d}\left|\mathbf{h}_{r, l}^{H} \boldsymbol{\Theta} \mathbf{h}_{l, r}+h_{l}\right|^{2}\right\}}{\mathbb{E}\left\{\sum_{m \in \mathcal{M}} x_{m, l} P_{m}^{c}\left|\mathbf{h}_{r, l}^{H} \boldsymbol{\Theta} \mathbf{h}_{m, r}+h_{m, l}\right|^{2}+\sigma^{2}\right\}}\right) \\
& =\log _{2}\left(1+\frac{P_{l}^{d} \mathbb{E}\left\{\left|\mathbf{h}_{r, l}^{H} \Theta \mathbf{h}_{l, r}+h_{l}\right|^{2}\right\}}{\sum_{m \in \mathcal{M}} x_{m, l} P_{m}^{c} \mathbb{E}\left\{\left|\mathbf{h}_{r, l}^{H} \boldsymbol{\Theta} \mathbf{h}_{m, r}+h_{m, l}\right|^{2}\right\}+\sigma^{2}}\right)
\end{aligned}
$$

where (a) follows from [29, Lemma 1].

Remark 1: Note that the approximation in (10) does not require the random variables in the numerator and denominator to be independent and becomes more accurate as the dimension of the channel matrix associated with the number of RIS reflecting elements increases. Thus, in our considered RIS-aided system, due to the large number of RIS reflecting elements, this approximation is usually accurate.

Next, we focus our attention on the computation of $\mathbb{E}\left\{\left|\mathbf{h}_{r, l}^{H} \boldsymbol{\Theta} \mathbf{h}_{l, r}+h_{l}\right|^{2}\right\}$ and $\mathbb{E}\left\{\left|\mathbf{h}_{r, l}^{H} \boldsymbol{\Theta} \mathbf{h}_{m, r}+h_{m, l}\right|^{2}\right\}$. Since it

$$
\begin{aligned}
\mathbb{E}\left\{\left|\mathbf{h}_{r, l}^{H} \Theta \mathbf{h}_{l, r}+h_{l}\right|^{2}\right\} & =\mathbb{E}\left\{\left|\left(\sqrt{\frac{\kappa_{r, l}}{1+\kappa_{r, l}}} \mathbf{h}_{r, l}^{\mathrm{LoS}}+\sqrt{\frac{1}{1+\kappa_{r, l}}} \mathbf{h}_{r, l}^{\mathrm{NLoS}}\right)^{H} \Theta\left(\sqrt{\frac{\kappa_{l, r}}{1+\kappa_{l, r}}} \mathbf{h}_{l, r}^{\mathrm{LoS}}+\sqrt{\frac{1}{1+\kappa_{l, r}}} \mathbf{h}_{l, r}^{\mathrm{NLoS}}\right)+h_{l}\right|^{2}\right\} \\
& =\mathbb{E}\left\{\left|h_{l}\right|^{2}\right\}+\Gamma_{1}\left(\left|\mathbf{x}_{1}\right|^{2}+\mathbb{E}\left\{\left|\mathbf{x}_{2}\right|^{2}\right\}+\mathbb{E}\left\{\left|\mathbf{x}_{3}\right|^{2}\right\}+\mathbb{E}\left\{\left|\mathbf{x}_{4}\right|^{2}\right\}\right) .
\end{aligned}
$$


is assumed that $\mathbf{h}_{l, r}, \mathbf{h}_{r, l}$, and $h_{l}$ are independent of each other, we obtain (11), shown at the bottom of the previous page, where

$$
\begin{aligned}
& \mathbf{x}_{1} \triangleq \sqrt{\kappa_{r, l} \kappa_{l, r}}\left(\mathbf{h}_{l, r}^{\mathrm{LoS}}\right)^{H} \boldsymbol{\Theta} \mathbf{h}_{r, l}^{\mathrm{LoS}}, \\
& \mathbf{x}_{2} \triangleq \sqrt{\kappa_{l, r}}\left(\mathbf{h}_{r, l}^{\mathrm{NLoS}}\right)^{H} \boldsymbol{\Theta} \mathbf{h}_{l, r}^{\mathrm{LoS}}, \\
& \mathbf{x}_{3} \triangleq \sqrt{\kappa_{l, r}}\left(\mathbf{h}_{r, l}^{\mathrm{NLoS}}\right)^{H} \boldsymbol{\Theta} \mathbf{h}_{l, r}^{\mathrm{LoS}}, \\
& \mathbf{x}_{4} \triangleq\left(\mathbf{h}_{r, l}^{\mathrm{NLoS}}\right)^{H} \boldsymbol{\Theta} \mathbf{h}_{l, r}^{\mathrm{NLoS}},
\end{aligned}
$$

and $\Gamma_{1} \triangleq \frac{1}{\left(1+\kappa_{r, l}\right)\left(1+\kappa_{l, r}\right)}$. Then, as far as the direct channel of the V2V link is concerned, it holds that $\mathbb{E}\left\{\left|h_{l}\right|^{2}\right\}=1$ (due to $h_{l} \sim \mathcal{C N}(0,1)$, and $\left|h_{l}\right|^{2} \sim \frac{1}{2} \chi^{2}(2)$ [30]). As for the desired cascaded channels, it holds that $\mathbb{E}\left\{\left|\mathbf{x}_{2}\right|^{2}\right\}=N \kappa_{r, l}$, $\mathbb{E}\left\{\left|\mathbf{x}_{3}\right|^{2}\right\}=N \kappa_{l, r}$, and $\mathbb{E}\left\{\left|\mathbf{x}_{4}\right|^{2}\right\}=N$. By inserting the results into (11), we obtain (13), shown at the bottom of the page.

Along the same lines, we can compute $\mathbb{E}\left\{\left|\mathbf{h}_{r, l}^{H} \boldsymbol{\Theta} \mathbf{h}_{m, r}+h_{m, l}\right|^{2}\right\}$. In particular, due to the fact that $\mathbf{h}_{m, r}, \mathbf{h}_{r, l}$, and $h_{m, l}$ are assumed to be independent of each other, we obtain (14), shown at the bottom of the page, where $\Gamma_{2}=\frac{1}{\left(1+\kappa_{r}\right)\left(1+\kappa_{m, r}\right)}$. By substituting (13) and (14) into (10), we have (15), shown at the bottom of the page.

Thus, a tractable and accurate approximation for the outage probability of $\mathrm{V} 2 \mathrm{~V}$ links is given by

$$
\begin{aligned}
& \operatorname{Pr}\left\{\log _{2}\left(1+\operatorname{SINR}_{l}\right) \leq R^{\text {th }}\right\} \\
& \quad=\mathbb{E}\left\{u\left(R^{\text {th }}-\log _{2}\left(1+\operatorname{SINR}_{l}\right)\right)\right\} \\
& \quad \approx \hat{u}_{\delta}\left(R^{\text {th }}-\log _{2}\left(1+\tilde{\gamma}_{l}(\mathbf{P}, \boldsymbol{\Theta}, \mathbf{X})\right)\right),
\end{aligned}
$$

where

$$
=\frac{\tilde{\gamma}_{l}(\mathbf{P}, \boldsymbol{\Theta}, \mathbf{X})}{\sum_{l}^{d}\left[\kappa_{r, l} \kappa_{l, r} \Gamma_{1}\left|\left(\mathbf{h}_{l, r}^{\mathrm{LoS}}\right)^{H} \boldsymbol{\Theta}_{m, l}^{\mathrm{LoS}}\right|^{2}+\xi_{1} P_{m}^{c}\left[\kappa_{r, l} \kappa_{m, r} \Gamma_{2}\left|\left(\mathbf{h}_{r, l}^{\mathrm{LoS}}\right)^{H} \boldsymbol{\Theta} \mathbf{h}_{m, r}^{\mathrm{LoS}}\right|^{2}+\xi_{2}\right]+\sigma^{2}\right.},
$$

$\xi_{1}=\Gamma_{1}\left(N \kappa_{r, l}+N \kappa_{l, r}+N\right)+1, \quad$ and $\quad \xi_{2}=$ $\Gamma_{2}\left(N \kappa_{r, l}+N \kappa_{m, r}+N\right)+1$. Furthermore, the constraint in (7b) can be rewritten as follows

$$
\hat{u}_{\delta}\left(R^{\mathrm{th}}-\log _{2}\left(1+\tilde{\gamma}_{l}(\mathbf{P}, \boldsymbol{\Theta}, \mathbf{X})\right)\right) \leq P_{\max }^{\text {outage }} .
$$

A more explicit form of (18) is given by

$$
\begin{aligned}
\log _{2}(1+\tilde{\gamma}(\mathbf{P}, \boldsymbol{\Theta}, \mathbf{X})) & \geq R^{\text {th }}+\delta^{-1} \ln \left(1 / P_{\max }^{\text {outage }}-1\right), \\
\Rightarrow \tilde{\gamma}_{l}(\mathbf{P}, \boldsymbol{\Theta}, \mathbf{X}) & \geq 2^{R^{\text {th }}+\delta^{-1} \ln \left(1 / P_{\max }^{\text {outage }}-1\right)}-1, \\
& \triangleq \tilde{\gamma}^{\text {const }},
\end{aligned}
$$

which is conveniently formulated in order to tackle the optimization problem in (7), as elaborated in the next section.

\section{Proposed Resource Allocation Algorithm}

To make the optimization problem in (7) more tractable, we first relax the binary variables in (7f) into continuous variables, which yields the following problem

$$
\begin{aligned}
\max _{\{\mathbf{F}, \boldsymbol{\Theta}, \mathbf{P}, \mathbf{X}\}} \sum_{m \in \mathcal{M}} R_{m} \\
\text { s.t. } 0 \leq x_{m, l} \leq 1, \forall m, l, \\
(7 \mathrm{c})-(7 \mathrm{e}),(19) .
\end{aligned}
$$

Such a relaxation usually implies that the objective value of problem (20) provides an upper bound for the objective value of problem (7). In problem (20), there are four optimization variables, i.e., the transmit power $\mathbf{P}$, the spectrum sharing strategy for V2V links $\mathbf{X}$, the MUD matrix $\mathbf{F}$, and the RIS reflection-coefficient matrix $\Theta$. Due to the coupling of these variables, there exists, in general, no standard method to solve this non-convex problem and it is prohibitive to find the globally optimal solution. Inspired by the wide applicability and efficiency of the BCD method, problem (20) is decomposed into three sub-problems that allows us to tackle the coupling of the optimization variables. More specifically, we alternately solve the problem for $\{\mathbf{P}, \mathbf{X}\}, \mathbf{F}$ and $\boldsymbol{\Theta}$ while fixing the other variables. This yields an altenating and iterative algorithm to obtain the near global optimal solution.

\section{A. Joint Optimization of Transmit Power and Spectrum Sharing}

For any given MUD matrix $\mathbf{F}$ and RIS reflection-coefficient matrix $\Theta$, the joint optimization of the transmit power $\mathbf{P}$ and spectrum sharing variables $\mathbf{X}$ can be formulated as the following problem

$$
\begin{aligned}
& \max _{\{\mathbf{P}, \mathbf{X}\}} \sum_{m \in \mathcal{M}} R_{m} \\
& \text { s.t. }(7 \mathrm{~d})-(7 \mathrm{e}),(19),(20 \mathrm{~b}) .
\end{aligned}
$$

$$
\begin{aligned}
\mathbb{E}\left\{\left|\mathbf{h}_{l, r}^{H} \boldsymbol{\Theta} \mathbf{h}_{r, l}+h_{m, l}\right|^{2}\right\} & =\kappa_{r, l} \kappa_{l, r} \Gamma_{1}\left|\left(\mathbf{h}_{l, r}^{\mathrm{LoS}}\right)^{H} \boldsymbol{\Theta} \mathbf{h}_{r, l}^{\mathrm{LoS}}\right|^{2}+\Gamma_{1}\left(N \kappa_{r, l}+N \kappa_{l, r}+N\right)+1 . \\
\mathbb{E}\left\{\left|\mathbf{h}_{r, l}^{H} \boldsymbol{\Theta} \mathbf{h}_{m, r}+h_{m, l}\right|^{2}\right\} & =\kappa_{r, l} \kappa_{m, r} \Gamma_{2}\left|\left(\mathbf{h}_{r, l}^{\mathrm{LoS}}\right)^{H} \boldsymbol{\Theta} \mathbf{h}_{m, r}^{\mathrm{LoS}}\right|^{2}+\Gamma_{2}\left(N \kappa_{r, l}+N \kappa_{m, r}+N\right)+1 . \\
\mathbb{E}\left\{\log _{2}\left(1+\mathrm{SINR}_{l}\right)\right\} & \leq \log _{2}\left(1+\frac{P_{l}^{d}\left[\kappa_{r, l} \kappa_{l, r} \Gamma_{1}\left|\left(\mathbf{h}_{l, r}^{\mathrm{LoS}}\right)^{H} \boldsymbol{\Theta} \mathbf{h}_{r, l}^{\mathrm{LoS}}\right|^{2}+\Gamma_{1}\left(N \kappa_{r, l}+N \kappa_{l, r}+N\right)+1\right]}{\left.\sum_{m \in \mathcal{M}} x_{m, l} P_{m}^{c}\left[\kappa_{r, l} \kappa_{m, r} \Gamma_{2}\left|\left(\mathbf{h}_{r, l}^{\mathrm{LoS}}\right)^{H} \boldsymbol{\Theta} \mathbf{h}_{m, r}^{\mathrm{LoS}}\right|^{2}+\Gamma_{2}\left(N \kappa_{r, l}+N \kappa_{m, r}+N\right)+1\right]+\sigma^{2}\right)} .\right.
\end{aligned}
$$


Problem (21) is neither a concave nor a quasi-concave maximization problem due to the non-concave objective function and non-convex constraints in (19). To deal with the bilinear product term $\sum_{m \in \mathcal{M}} x_{m, l} P_{m}^{c}$ in constraint (19), we invoke the $\log$-exponential reformulation method, i.e., we write $P_{m}^{c}=$ $\exp \left(\tilde{P}_{m}^{c}\right), P_{l}^{d}=\exp \left(\tilde{P}_{l}^{d}\right)$ and $x_{m, l}=\exp \left(\tilde{x}_{m, l}\right)$. In addition, the successive convex approximation (SCA) technique is utilized to approximate the original function with a more tractable function at some given local points in each iteration. Then, we define $\tilde{\mathbf{P}}^{(r)}=\left\{\tilde{P}_{m}^{c,(r)}, \tilde{P}_{l}^{d,(r)}, \forall m, l\right\}$ and $\tilde{\mathbf{X}}^{(r)}=$ $\left\{\tilde{x}_{m, l}^{(r)}, \forall m, l\right\}$ as the given transmit power and spectrum sharing variables at the $r$ th iteration. Moreover, we observe that $-R_{m}$ has the form $f(x, y, z)=\log _{2}\left(a e^{x}+b e^{y+z}+c\right)-$ $\log _{2}\left(b e^{y+z}+c\right)$, where $a, b, c$ are all positive constants, and $x, y, z \geq 0$. Since both $f_{1}(x, y, z)$ and $f_{2}(y, z)$ have the form of "log-sum-exp", in light of [31, Sec. 3.1.4], $f_{1}(x, y, z)$ is jointly convex with respect to $x, y$ and $z$, and $f_{2}(y, z)$ is jointly convex with respect to $y$ and $z$.

Thus, $-R_{m}$ can be further written as the difference of two convex functions. Owing to the fact that any convex function is globally lower-bounded by its first-order Taylor expansion at any point [32], at some given local points $\tilde{\mathbf{X}}^{(r)}$ and $\tilde{\mathbf{P}}^{(r)}$, we have

$$
\begin{aligned}
-R_{m}= & \log _{2}\left(\sum_{l \in \mathcal{L}} C_{2} \exp \left(\tilde{x}_{m, l}+\tilde{P}_{l}^{d}\right)+\sigma^{2}\left|\mathbf{f}_{m}^{H}\right|^{2}\right) \\
& -\log _{2}\left(C_{1} \exp \left(\tilde{P}_{m}^{c}\right)+\sum_{l \in \mathcal{L}} C_{2} \exp \left(\tilde{x}_{m, l}+\tilde{P}_{l}^{d}\right)\right. \\
& \left.+\sigma^{2}\left|\mathbf{f}_{m}^{H}\right|^{2}\right) \\
\leq & \log _{2}\left(\sum_{l \in \mathcal{L}} C_{2} \exp \left(\tilde{x}_{m, l}+\tilde{P}_{l}^{d}\right)+\sigma^{2}\left|\mathbf{f}_{m}^{H}\right|^{2}\right)-\tilde{R}_{m} \\
\triangleq & R_{m}^{u b},
\end{aligned}
$$

where $\tilde{R}_{m}$ is given by

$$
\begin{aligned}
\tilde{R}_{m}= & \log _{2}\left[C_{1} \exp \left(\tilde{P}_{m}^{c,(r)}\right)+\sum_{l \in \mathcal{L}} C_{2} \exp \left(\tilde{x}_{m, l}^{(r)}+\tilde{P}_{l}^{d,(r)}\right)\right. \\
& \left.+\sigma^{2}\left|\mathbf{f}_{m}^{H}\right|^{2}\right]+\Delta_{1}\left(\tilde{P}_{m}^{c}-\tilde{P}_{m}^{c,(r)}\right) \\
& +\sum_{l \in \mathcal{L}}\left(\Delta_{2}\left(\tilde{x}_{m, l}-\tilde{x}_{m, l}^{(r)}\right)+\Delta_{3}\left(\tilde{P}_{l}^{d}-\tilde{P}_{l}^{d,(r)}\right)\right),
\end{aligned}
$$

and $C_{1}=\left|\mathbf{f}_{m}^{H}\left(\mathbf{h}_{r, b} \Theta \mathbf{h}_{m, r}+\mathbf{h}_{m, b}\right)\right|^{2}, C_{2}=\mid \mathbf{f}_{m}^{H}\left(\mathbf{h}_{r, b}\right.$ $\left.\boldsymbol{\Theta h}_{l, r}+\mathbf{h}_{l, b}\right)\left.\right|^{2}$. The coefficients $\Delta_{1}, \Delta_{2}$ and $\Delta_{3}$ in (23) are constant terms related to the derivative, which are omitted here for brevity. Hence, we obtain an upper bound for $-R_{m}$, i.e., $R_{m}^{u b}$, which is a convex function. After variables mapping, the constraint in (19) is equivalent to,

$$
\begin{aligned}
& \tilde{\gamma}^{\text {const }}\left(\sum_{m \in \mathcal{M}}\left(\kappa_{r, l} \kappa_{m, r} \Gamma_{2}\left|\left(\mathbf{h}_{r, l}^{\mathrm{LoS}}\right)^{H} \boldsymbol{\Theta} \mathbf{h}_{m, r}^{\mathrm{LoS}}\right|^{2}+\xi_{2}\right)\right. \\
& \left.\quad \times \exp \left(\tilde{x}_{m, l}+\tilde{P}_{m}^{c}\right)+\sigma^{2}\right) \\
& \leq\left(\kappa_{r, l} \kappa_{l, r} \Gamma_{1}\left|\left(\mathbf{h}_{l, r}^{\mathrm{LoS}}\right)^{H} \boldsymbol{\Theta} \mathbf{h}_{r, l}^{\mathrm{LoS}}\right|^{2}+\xi_{1}\right) \exp \left(\tilde{P}_{l}^{d}\right),
\end{aligned}
$$

which is not a convex constraint. By applying the first-order Taylor expansion at the given point $\tilde{\mathbf{P}}^{(r)}$ to $\exp \left(\tilde{P}_{l}^{d}\right)$, we obtain

$$
\begin{gathered}
\exp \left(\tilde{P}_{l}^{d}\right) \geq \exp \left(\tilde{P}_{l}^{d,(r)}\right)+\exp \left(\tilde{P}_{l}^{d,(r)}\right)\left(\tilde{P}_{l}^{d}-\tilde{P}_{l}^{d,(r)}\right) \\
=\Lambda_{l}^{l b} .
\end{gathered}
$$

Then, (24) can be approximated with the convex constraint as follows

$$
\begin{aligned}
\tilde{\gamma}^{\text {const }} & \left(\sum_{m \in \mathcal{M}}\left(\kappa_{r, l} \kappa_{m, r} \Gamma_{2}\left|\left(\mathbf{h}_{r, l}^{\mathrm{LoS}}\right)^{H} \boldsymbol{\Theta} \mathbf{h}_{m, r}^{\mathrm{LoS}}\right|^{2}+\xi_{2}\right)\right. \\
& \left.\times \exp \left(\tilde{x}_{m, l}+\tilde{P}_{m}^{c}\right)+\sigma^{2}\right) \\
\leq & \left(\kappa_{r, l} \kappa_{l, r} \Gamma_{1}\left|\left(\mathbf{h}_{l, r}^{\mathrm{LoS}}\right)^{H} \boldsymbol{\Theta} \mathbf{h}_{r, l}^{\mathrm{LoS}}\right|^{2}+\xi_{1}\right) \Lambda_{l}^{l b} .
\end{aligned}
$$

Therefore, at the given local points $\tilde{\mathbf{X}}^{(r)}$ and $\tilde{\mathbf{P}}^{(r)}$, problem (21) is reformulated as

$$
\begin{aligned}
& \min _{\{\tilde{\mathbf{P}}, \tilde{\mathbf{x}}\}} \sum_{m \in \mathcal{M}} R_{m}^{u b} \\
& \text { s.t. } 0 \leq \exp \left(\tilde{P}_{m}^{c}\right) \leq P_{\max }^{c}, \forall m, \\
& 0 \leq \exp \left(\tilde{P}_{l}^{d}\right) \leq P_{\max }^{d}, \forall l \\
& \sum_{l \in \mathcal{L}} \exp \left(\tilde{x}_{m, l}\right) \leq 1, \forall m, \\
& \sum_{m \in \mathcal{M}} \exp \left(\tilde{x}_{m, l}\right) \leq 1, \forall l, \\
& 0 \leq \exp \left(\tilde{x}_{m, l}\right) \leq 1, \forall m, l
\end{aligned}
$$

(26).

Since the constraints (27b)-(27e) and the objective function are convex, problem (27) is a convex optimization problem that can be efficiently solved by using standard convex optimization solvers, such as CVX [33] and MOSEK [34]. It is worth noting that any feasible solution to problem (27) is still feasible for problem (21), but the reverse does not hold in general. Therefore, the absolute of the optimal objective value obtained by the approximated problem (27) provides a lower bound for the optimal objective value of problem (21).

\section{B. Design of the MUD Matrix}

For fixed spectrum sharing variables $\mathbf{X}$, transmit power $\mathbf{P}$, and RIS reflection coefficient matrix $\Theta$, the MUD matrix $\mathbf{F}$ can be designed by leveraging the SDR method. Define $\mathbf{V}_{m}=\mathbf{f}_{m} \mathbf{f}_{m}^{H}, \forall m$, with the rank constraint Rank 
$\left(\mathbf{V}_{m}\right) \leq 1, \forall m$. For simplicity, denote $\mathbf{h}_{m}=\mathbf{h}_{r, b} \Theta \mathbf{h}_{m, r}+$ $\mathbf{h}_{m, b}, \overline{\mathbf{h}}_{l}=\mathbf{h}_{r, b} \Theta \mathbf{h}_{l, r}+\mathbf{h}_{l, b}$, and define $\mathbf{H}_{m}=\mathbf{h}_{m} \mathbf{h}_{m}^{H}$, $\mathbf{H}_{l}=\mathbf{h}_{l} \mathbf{h}_{l}^{H}$. Then, the optimization problem can be formulated as follows

$\max _{\left\{\mathbf{V}_{m}\right\}} \sum_{m \in \mathcal{M}} \log _{2}\left(1+\frac{P_{m}^{c} \operatorname{Tr}\left(\mathbf{H}_{m} \mathbf{V}_{m}\right)}{\sum_{l \in \mathcal{L}} x_{m, l} P_{l}^{d} \operatorname{Tr}\left(\mathbf{H}_{l} \mathbf{V}_{m}\right)+\sigma^{2} \operatorname{Tr}\left(\mathbf{V}_{m}\right)}\right)$

s.t. $\operatorname{Rank}\left(\mathbf{V}_{m}\right) \leq 1, \forall m$,

$$
\mathbf{V}_{m} \succeq 0, \forall m \text {. }
$$

Owing to the non-concavity of the objective function and the rank-one constraint in (28b), problem (28) is non-convex. To tackle this problem, we first ignore the rank-one constraint in (28b) for all variables $\mathbf{V}_{m}$, and then exploit the SCA technique to approximate the objective function with a more tractable function in each iteration. Furthermore, the objective function can be written as the difference between two concave functions, and due to the fact that any concave function is globally upper-bounded by its first-order Taylor expansion at any point [32], at the given local point $\mathbf{V}_{m}^{(r)}$ we have

$$
\begin{aligned}
\log _{2}\left(1+\frac{P_{m}^{c} \operatorname{Tr}\left(\mathbf{H}_{m} \mathbf{V}_{m}\right)}{\sum_{l \in \mathcal{L}} x_{m, l} P_{l}^{d} \operatorname{Tr}\left(\mathbf{H}_{l} \mathbf{V}_{m}\right)+\sigma^{2} \operatorname{Tr}\left(\mathbf{V}_{m}\right)}\right) \\
=\log _{2}\left(P_{m}^{c} \operatorname{Tr}\left(\mathbf{H}_{m} \mathbf{V}_{m}\right)+\sum_{l \in \mathcal{L}} x_{m, l} P_{l}^{d} \operatorname{Tr}\left(\mathbf{H}_{l} \mathbf{V}_{m}\right)\right. \\
\left.\quad+\sigma^{2} \operatorname{Tr}\left(\mathbf{V}_{m}\right)\right) \\
\quad-\log _{2}\left(\sum_{l \in \mathcal{L}} x_{m, l} P_{l}^{d} \operatorname{Tr}\left(\mathbf{H}_{l} \mathbf{V}_{m}\right)+\sigma^{2} \operatorname{Tr}\left(\mathbf{V}_{m}\right)\right) \\
\geq \log _{2}\left(P_{m}^{c} \operatorname{Tr}\left(\mathbf{H}_{m} \mathbf{V}_{m}\right)+\sum_{l \in \mathcal{L}} x_{m, l} P_{l}^{d} \operatorname{Tr}\left(\mathbf{H}_{l} \mathbf{V}_{m}\right)\right. \\
\left.\quad+\sigma^{2} \operatorname{Tr}\left(\mathbf{V}_{m}\right)\right)-\tilde{R}_{m}^{u b \triangleq} \tilde{R}_{m}^{l b},
\end{aligned}
$$

where

$$
\begin{gathered}
\tilde{R}_{m}^{u b}=\log _{2}\left(\sum_{l \in \mathcal{L}} x_{m, l} P_{l}^{d} \operatorname{Tr}\left(\mathbf{H}_{l} \mathbf{V}_{m}^{(r)}\right)+\sigma^{2} \operatorname{Tr}\left(\mathbf{V}_{m}^{(r)}\right)\right) \\
+\Omega_{m}^{-1} \operatorname{Tr}\left(\left(\sum_{l \in \mathcal{L}} x_{m, l} P_{l}^{d} \mathbf{H}_{l}+\sigma^{2} \mathbf{I}_{K}\right)\left(\mathbf{V}_{m}-\mathbf{V}_{m}^{(r)}\right)\right),
\end{gathered}
$$

and $\Omega_{m}=\left(\sum_{l \in \mathcal{L}} x_{m, l} P_{l}^{d} \operatorname{Tr}\left(\mathbf{H}_{l} \mathbf{V}_{m}^{(r)}\right)+\sigma^{2} \operatorname{Tr}\left(\mathbf{V}_{m}^{(r)}\right)\right) \ln 2$. Thus, problem (28) can be approximated with the following problem

$$
\begin{aligned}
& \max _{\left\{\mathbf{V}_{m}\right\}} \sum_{m \in \mathcal{M}} \tilde{R}_{m}^{l b} \\
& \text { s.t. } \mathbf{V}_{m} \succeq 0, \forall m .
\end{aligned}
$$

By direct inspection, we observe that problem (31) is convex. If $\left\{\mathbf{V}_{m}^{*}\right\}$ satisfies the constraint $\operatorname{Rank}\left(\mathbf{V}_{m}^{*}\right)=1, \forall m$, the optimal $\mathbf{f}_{m}^{*}$ to problem (31) can be obtained from the eigenvalue decomposition (EVD) of $\mathbf{V}_{m}^{*}$. If $\operatorname{Rank}\left(\mathbf{V}_{m}^{*}\right)>1$, on the other hand, problem (28) and (31) are not necessarily equivalent, which implies that the solution obtained by (31) is not feasible to problem (28). In the following theorem, we show that the solution to problem (31) satisfies the constraint $\operatorname{Rank}\left(\mathbf{V}_{m}^{*}\right)=1, \forall m$, which implies that the SDR method is tight.

Theorem 1: The solution of problem (31) is an optimal MUD matrix $\left\{\mathbf{V}_{m}^{*}\right\}$ that satisfies the constraint Rank $\left(\mathbf{V}_{m}^{*}\right)=1, \forall m$.

Proof: See Appendix A.

Theorem 1 indicates that the rank relaxation on $\mathbf{V}_{m}$ in problem (31) results in no loss of optimality to problem (28). Thus, problem (31) can be efficiently solved by using CVX [33]. It is worth noting that the lower bound adopted in (29) suggests that any feasible solution of problem (31) is also feasible for problem (28). As a result, the optimal objective value obtained from the approximate problem (31) provides a lower bound for the objective value of problem (31).

\section{Optimization of the RIS Reflection Coefficients}

For the given transmit power $\mathbf{P}$, MUD matrix $\mathbf{F}$ and spectrum sharing $\mathbf{X}$, the RIS reflection-coefficient matrix $\Theta$ is optimized in this subsection. As for the V2I links, we denote by $\mathbf{h}_{m}^{c}=\mathbf{h}_{r, b} \operatorname{diag}\left\{\mathbf{h}_{m, r}\right\} \in \mathbb{C}^{K \times N}$ the cascaded desired channel from the $m$ th $\mathrm{C}$-VUE to the BS via the RIS, and by $\mathbf{h}_{l}^{c}=\mathbf{h}_{r, b} \operatorname{diag}\left\{\mathbf{h}_{l, r}\right\} \in \mathbb{C}^{K \times N}$ the cascaded crosstalk channel from the transmitter of the $l$ th D2D-V2V to the BS via the RIS. The superscript "c" denotes "cascaded". As for the V2V links, we denote by $\mathbf{G}_{l}=\operatorname{diag}\left\{\mathbf{h}_{r, l}\right\} \mathbf{h}_{l, r} \in \mathbb{C}^{N \times 1}$ the cascaded desired channel between the $l$ th $\mathrm{D} 2 \mathrm{D}-\mathrm{V} 2 \mathrm{~V}$ pair via the RIS, and by $\mathbf{G}_{m, l}=\operatorname{diag}\left\{\mathbf{h}_{r, l}\right\} \mathbf{h}_{m, r} \in \mathbb{C}^{N \times 1}$ the cascaded crosstalk channel from the $m$ th $\mathrm{C}$-VUE to the receiver of the $l$ th D2D-V2V via the RIS. Let $\boldsymbol{\theta}=\left[\theta_{1}, \theta_{2}, \ldots, \theta_{N}\right]^{T} \in \mathbb{C}^{N \times 1}$ be the vector containing the diagonal elements of matrix $\Theta$. Thus, $\operatorname{SINR}_{m}$ and $\tilde{\gamma}_{l}$ (defined in (17)) can be reformulated as

$$
\begin{aligned}
& \operatorname{SINR}_{m} \\
& =\frac{P_{m}^{c}\left|\mathbf{f}_{m}^{H}\left(\mathbf{h}_{m}^{c} \boldsymbol{\theta}+\mathbf{h}_{m, b}\right)\right|^{2}}{\sum_{l \in \mathcal{L}} x_{m, l} P_{l}^{d}\left|\mathbf{f}_{m}^{H}\left(\mathbf{h}_{l}^{c} \boldsymbol{\theta}+\mathbf{h}_{l, b}\right)\right|^{2}+\sigma^{2}\left|\mathbf{f}_{m}^{H}\right|^{2}}, \\
& \tilde{\gamma}_{l}=\frac{P_{l}^{d}\left(\kappa_{r, l} \kappa_{l, r} \Gamma_{1}\left|\left(\mathbf{G}_{l}^{\mathrm{LoS}}\right)^{H} \boldsymbol{\theta}\right|^{2}+\xi_{1}\right)}{\sum_{m \in \mathcal{M}} x_{m, l} P_{m}^{c}\left(\kappa_{r, l} \kappa_{m, r} \Gamma_{2}\left|\left(\mathbf{G}_{m, l}^{\mathrm{LoS}}\right)^{H} \boldsymbol{\theta}\right|^{2}+\xi_{2}\right)+\sigma^{2}},
\end{aligned}
$$

respectively, which yields the following problem

$$
\begin{aligned}
& \max _{\{\boldsymbol{\theta}\}} \sum_{m \in \mathcal{M}} \log _{2}\left(1+\operatorname{SINR}_{m}(\boldsymbol{\theta})\right) \\
& \text { s.t. } \tilde{\gamma}_{l}(\boldsymbol{\theta}) \geq \tilde{\gamma}^{\mathrm{const}}, \forall l \\
& \quad\left|\theta_{n}\right|=1, \forall n .
\end{aligned}
$$


The obstacles for solving problem (34) are the non-concavity of the objective function, the non-convexity of the constraint (34b) and the unit-modulus constraint in (34c). To cope with these issues, we capitalize on the penalty CCP method [35]. We note, in particular, that the SDR method, utilized in the previous subsection, is a very straightforward idea to design RIS reflection coefficients. However, the solution obtained by SDR may be undesirable provided that the initial points drops outside of the feasible region constituted by constraints (34b) and (34c). It is prohibitive to obtain such ideal initial points. In this regard, the penalty CCP method is a favorable approach even if an infeasible initial point is adopted. Furthermore, the pivotal thought of the penalty CCP method is to relax the feasible region by adding slack variables to constraints. Such a relaxation can be viewed as a violation to the original region, and the violation needs to be penalized in the objective function. By initially putting a low penalty on violations, the constraints are allowed to be violated so that a region with lower objective value can be found, which, thus, is applicable for a wider range of situations. Next, we introduce the application of penalty CCP method to solve problem (34).

To solve the problem in (34), the objective function, which can be written as the difference of two log-functions, is approximated with a concave function by using the first-order Taylor expansion at any given points $\boldsymbol{\theta}^{(i)}$ as shown in (35a), shown at the bottom of the page. The constraint in (34b) can be reformulated as

$$
\begin{gathered}
\tilde{\gamma}^{\text {const }}\left(\sum_{m \in \mathcal{M}} x_{m, l} P_{m}^{c}\left(\kappa_{r, l} \kappa_{m, r} \Gamma_{2}\left|\left(\mathbf{G}_{m, l}^{\mathrm{LoS}}\right)^{H} \boldsymbol{\theta}\right|^{2}+\xi_{2}\right)+\sigma^{2}\right) \\
\leq \underbrace{P_{l}^{d}\left(\kappa_{r, l} \kappa_{l, r} \Gamma_{1}\left|\left(\mathbf{G}_{l}^{\mathrm{LoS}}\right)^{H} \boldsymbol{\theta}\right|^{2}+\xi_{1}\right)}_{f_{3}(\boldsymbol{\theta})}
\end{gathered}
$$

Due to the upper bound of $f_{3}(\boldsymbol{\theta})$ in (35d), shown at the bottom of the page, (34b) is further approximated as

$$
\begin{array}{r}
\tilde{\gamma}^{\text {const }}\left(\sum _ { m \in \mathcal { M } } x _ { m , l } P _ { m } ^ { c } \left(\kappa_{r, l} \kappa_{m, r} \Gamma_{2}\left|\left(\mathbf{G}_{m, l}^{\operatorname{LoS}}\right)^{H} \boldsymbol{\theta}\right|^{2}\right.\right. \\
\left.\left.+\xi_{2}\right)+\sigma^{2}\right) \leq \tilde{f}_{3}(\boldsymbol{\theta}),
\end{array}
$$

which is a convex constraint.

The unit-modulus constraint is equivalent to $1 \leq\left|\theta_{n}\right| \leq$ $1, \forall n$. The non-convex parts of the resulting constraint, at any given point $\boldsymbol{\theta}^{(i)}$, can be linearized as follows

$$
\left|\theta_{n}^{(i)}\right|^{2}-2 \Re\left\{\left(\theta_{n}^{*}\right)^{(i)} \theta_{n}\right\} \leq-1 .
$$

Eventually, we obtain the following convex subproblem for $\boldsymbol{\theta}$

$$
\begin{aligned}
& \max _{\{\boldsymbol{\theta}, \mathbf{b}\}} f_{1}(\boldsymbol{\theta})-\tilde{f}_{2}(\boldsymbol{\theta})-\lambda^{(i)} \sum_{b=1}^{2 N+1} b_{n} \\
& \text { s.t. }\left|\theta_{n}^{(i)}\right|^{2}-2 \Re\left\{\left(\theta_{n}^{*}\right)^{(i)} \theta_{n}\right\} \leq b_{n}-1, \forall n, \\
& \left|\theta_{n}\right| \leq 1+b_{N+n}, \forall n, \\
& \tilde{\gamma}^{\text {const }}\left(\sum_{m \in \mathcal{M}} x_{m, l} P_{m}^{c}\left(\kappa_{r, l} \kappa_{m, r} \Gamma_{2}\left|\left(\mathbf{G}_{m, l}^{\mathrm{LoS}}\right)^{H} \boldsymbol{\theta}\right|^{2}+\xi_{2}\right)\right. \\
& \left.+\sigma^{2}\right) \leq \tilde{f}_{3}(\boldsymbol{\theta})+b_{2 N+1}, \forall l,
\end{aligned}
$$

where $\mathbf{b}=\left[b_{1}, \ldots, b_{N}, \ldots, b_{2 N}, b_{2 N+1}\right]^{T}$ are the slack variables imposed over the associated constraints of the RIS reflection coefficients $\boldsymbol{\theta}$, and $\|\mathbf{b}\|_{1}$ is the penalty term of the objective function, which is scaled by the penalty factor $\lambda^{(q)}$ that enforces the violated constraints. Problem (34) is a convex problem that can be solved by off-the-shelf tools, such as CVX [33] and MOSEK [34]. The steps for finding a feasible $\boldsymbol{\theta}$ to problem (34) are summarized in Algorithm 1.

Remark 2: Several remarks on the proposed penalty CPP-based algorithm can be made: i) The constraints that are

$$
\begin{aligned}
& \underbrace{\sum_{m \in \mathcal{M}} \log _{2}\left(P_{m}^{c}\left|\mathbf{f}_{m}^{H}\left(\mathbf{h}_{m}^{c} \boldsymbol{\theta}+\mathbf{h}_{m, b}\right)\right|^{2}+\sum_{l \in \mathcal{L}} x_{m, l} P_{l}^{d}\left|\mathbf{f}_{m}^{H}\left(\mathbf{h}_{l}^{c} \boldsymbol{\theta}+\mathbf{h}_{l, b}\right)\right|^{2}+\sigma^{2}\left|\mathbf{f}_{m}^{H}\right|^{2}\right)}_{f_{1}(\boldsymbol{\theta})} \\
& -\underbrace{\sum_{m \in \mathcal{M}} \log _{2}\left(\sum_{l \in \mathcal{L}} x_{m, l} P_{l}^{d}\left|\mathbf{f}_{m}^{H}\left(\mathbf{h}_{l}^{c} \boldsymbol{\theta}+\mathbf{h}_{l, b}\right)\right|^{2}+\sigma^{2}\left|\mathbf{f}_{m}^{H}\right|^{2}\right)}_{f_{2}(\boldsymbol{\theta})} \\
& \geq f_{1}(\boldsymbol{\theta})-\left(f_{2}\left(\boldsymbol{\theta}^{(i)}\right)+\nabla_{\boldsymbol{\theta}} f_{2}\left(\boldsymbol{\theta}^{(i)}\right)^{T}\left(\boldsymbol{\theta}-\boldsymbol{\theta}^{(i)}\right)\right) \triangleq f_{1}(\boldsymbol{\theta})-\tilde{f}_{2}(\boldsymbol{\theta}), \\
& \nabla_{\boldsymbol{\theta}} f_{2}\left(\boldsymbol{\theta}^{(i)}\right)=\sum_{m \in \mathcal{M}} \frac{2}{B_{m}\left(\boldsymbol{\theta}^{(i)}\right) \ln 2}\left[\sum_{l \in \mathcal{L}} x_{m, l} P_{l}^{d}\left(\left(\mathbf{h}_{l}^{c}\right)^{H} \mathbf{f}_{m} \mathbf{f}_{m}^{H} \mathbf{h}_{l}^{c} \boldsymbol{\theta}^{(i)}+\left(\mathbf{h}_{l}^{c}\right)^{H} \mathbf{f}_{m} \mathbf{f}_{m}^{H} \mathbf{h}_{l, b}\right)\right], \\
& B_{m}\left(\boldsymbol{\theta}^{(i)}\right)=\sum_{l \in \mathcal{L}} x_{m, l} P_{l}^{d}\left|\mathbf{f}_{m}^{H}\left(\mathbf{h}_{l}^{c} \boldsymbol{\theta}^{(i)}+\mathbf{h}_{l, b}\right)\right|^{2}+\sigma^{2}\left|\mathbf{f}_{m}^{H}\right|^{2} . \\
& f_{3}(\boldsymbol{\theta}) \leq P_{l}^{d}\left[\left(\left|\left(\mathbf{G}_{l}^{\mathrm{LoS}}\right)^{H} \boldsymbol{\theta}^{(i)}\right|^{2}+\left(\boldsymbol{\theta}^{(i)}\right)^{H} \mathbf{G}_{l}^{\mathrm{LoS}}\left(\mathbf{G}_{l}^{\mathrm{LoS}}\right)^{H}\left(\boldsymbol{\theta}-\boldsymbol{\theta}^{(i)}\right)\right) \kappa_{r, l} \kappa_{l, r} \Gamma_{1}+\xi_{1}\right] \triangleq \tilde{f}_{3}(\boldsymbol{\theta}) .
\end{aligned}
$$




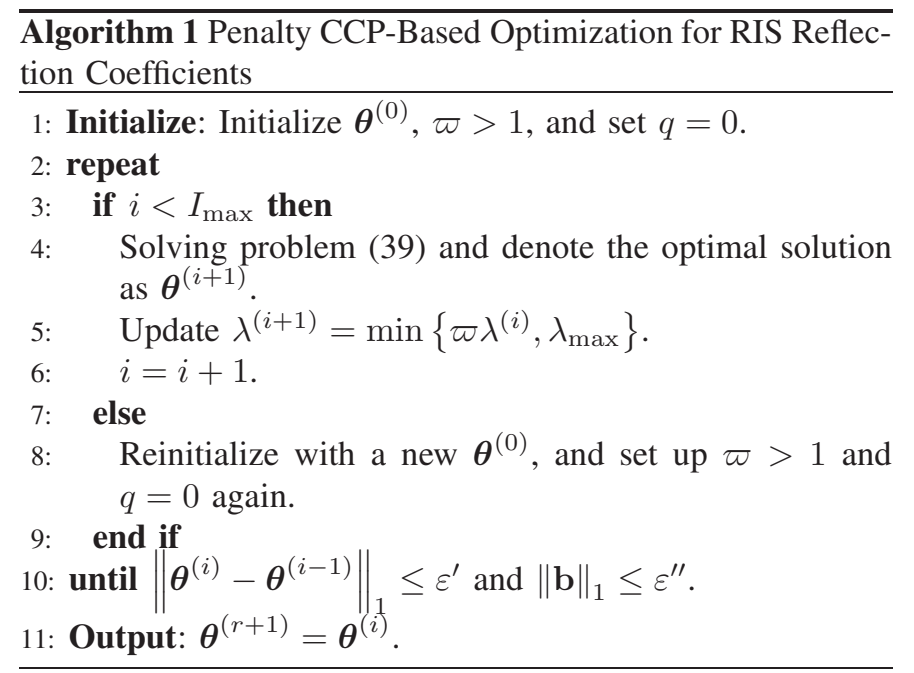

enforced with the slack variables expand the search area of the solution, and may lead to faster convergence and better numerical stability. The slack variables need to be sufficiently small to enforce the relaxed region to approach the region of the original problem. ii) The upper limit $\lambda_{\max }$ on $\lambda$ is imposed to avoid numerical problems. To be specific, a feasible solution satisfying $\|\mathbf{b}\|_{1} \leq \varepsilon^{\prime \prime}$ is not found when the iteration converges to the stopping criterion $\left\|\boldsymbol{\theta}^{(i)}-\boldsymbol{\theta}^{(i-1)}\right\|_{1} \leq \varepsilon^{\prime}$ as $\lambda^{(i)}$ increases, which implies that the stopping criteria $\left\|\boldsymbol{\theta}^{(i)}-\boldsymbol{\theta}^{(i-1)}\right\|_{1} \leq \varepsilon^{\prime}$ controls the convergence of Algorithm 1. iii) The penalty CCP-based algorithm is not a descent algorithm, but it still yields good convergence performance whose details can be found in [35].

\section{Proposed Algorithm, Convergence and Complexity}

Based on the above three subproblems, we propose an effective iterative algorithm to obtain a near optimal solution for problem (20) under the framework of the BCD algorithm. We optimize one of the decomposed block of variables while fixing the other variables in an alternating manner, until the convergence of the objective function is achieved, which yields the proposed AOIA algorithm. The details of the proposed AOIA are given in Algorithm 2. The convergence behavior of our proposed AOIA algorithm is analyzed in the following. Define $\Phi\left(\mathbf{P}^{(r)}, \mathbf{X}^{(r)}, \mathbf{F}^{(r)}, \boldsymbol{\Theta}^{(r)}\right)$ as the objective function of problem (20) at the $r$ th iteration. First, in Step 4 of Algorithm 2, problem (27) is optimized with given $\left\{\mathbf{F}^{(r)}, \Theta^{(r)}\right\}$, and we have

$$
\begin{aligned}
\Phi\left(\mathbf{P}^{(r)}, \mathbf{X}^{(r)},\right. & \left.\mathbf{F}^{(r)}, \mathbf{\Theta}^{(r)}\right) \stackrel{(a)}{=} \Phi_{\mathbf{P}, \mathbf{X}}^{u b}\left(\mathbf{P}^{(r)}, \mathbf{X}^{(r)}, \mathbf{F}^{(r)}, \Theta^{(r)}\right) \\
& \stackrel{(b)}{\geq} \Phi_{\mathbf{P}, \mathbf{X}}^{u b}\left(\mathbf{P}^{(r+1)}, \mathbf{X}^{(r+1)}, \mathbf{F}^{(r)}, \mathbf{\Theta}^{(r)}\right),
\end{aligned}
$$

where $\Phi_{\mathbf{P}, \mathbf{X}}^{u b}\left(\mathbf{P}^{(r)}, \mathbf{X}^{(r)}, \mathbf{F}^{(r)}, \boldsymbol{\Theta}^{(r)}\right)$ is the objective function of problem (27), and (a) follows from the fact that the first-order Taylor expansions are tight at the given local points of problem (27) and (b) holds due to the optimality of the solution of problem (27). Since the absolute value of the optimal objective value obtained by the approximated \begin{tabular}{lllll}
\hline Algorithm 2 & Alternating & Optimization-Based & Iterative
\end{tabular} Algorithm (AOIA)

1: Initialize $\mathbf{F}^{(0)}$ and $\Theta^{(0)}$, and set the iteration index $r=0$.

2: repeat

3: Solve problem (27) for given $\left\{\mathbf{F}^{(r)}, \boldsymbol{\Theta}^{(r)}\right\}$, and denote the optimal solution as $\left\{\mathbf{X}^{(r+1)}, \mathbf{P}^{(r+1)}\right\}$.

4: Solve problem (31) for given $\left\{\mathbf{X}^{(r+1)}, \mathbf{P}^{(r+1)}, \mathbf{F}^{(r)}, \Theta^{(r)}\right\}$, and denote the optimal solution as $\left\{\mathbf{F}^{(r+1)}\right\}$.

5: For given $\left\{\mathbf{X}^{(r+1)}, \mathbf{P}^{(r+1)}, \mathbf{F}^{(r+1)}, \boldsymbol{\Theta}^{(r)}\right\}$, obtain the optimal RIS reflection-coefficient matrix $\left\{\boldsymbol{\Theta}^{(r+1)}\right\}$ via Algorithm 1.

6: $r=r+1$.

7: until The change of the objective value is below a threshold $\epsilon>0$. Return the optimal solution $\left\{\mathbf{X}^{(*)}, \mathbf{P}^{(*)}, \mathbf{F}^{(*)}, \mathbf{\Theta}^{(*)}\right\}$.

problem (27) provides a lower bound of that of problem (21), we have

$$
\begin{aligned}
\left|\Phi_{\mathbf{P}, \mathbf{X}}^{u b}\left(\mathbf{P}^{(r+1)}, \mathbf{X}^{(r+1)}, \mathbf{F}^{(r)}, \boldsymbol{\Theta}^{(r)}\right)\right| \\
\quad \leq \Phi\left(\mathbf{P}^{(r+1)}, \mathbf{X}^{(r+1)}, \mathbf{F}^{(r)}, \Theta^{(r)}\right) .
\end{aligned}
$$

From the above inequality, we evince that, the objective value of problem (21) is non-increasing after each iteration.

Similarly, in Step 4 of Algorithm 2, it follows that

$$
\begin{aligned}
\Phi & \left(\mathbf{P}^{(r+1)}, \mathbf{X}^{(r+1)}, \mathbf{F}^{(r)}, \Theta^{(r)}\right) \\
& =\Phi_{\mathbf{F}}^{l b}\left(\mathbf{P}^{(r+1)}, \mathbf{X}^{(r+1)}, \mathbf{F}^{(r)}, \Theta^{(r)}\right) \\
& \leq \Phi_{\mathbf{F}}^{l b}\left(\mathbf{P}^{(r+1)}, \mathbf{X}^{(r+1)}, \mathbf{F}^{(r+1)}, \boldsymbol{\Theta}^{(r)}\right) \\
& \leq \Phi\left(\mathbf{P}^{(r+1)}, \mathbf{X}^{(r+1)}, \mathbf{F}^{(r+1)}, \mathbf{\Theta}^{(r)}\right)
\end{aligned}
$$

where $\Phi_{\mathbf{F}}^{l b}\left(\mathbf{P}^{(r+1)}, \mathbf{X}^{(r+1)}, \mathbf{F}^{(r)}, \Theta^{(r)}\right)$ represents the objective function of problem (31). In Step 5 of Algorithm 2, since problem (34) is solved optimally, it holds that $\Phi\left(\mathbf{P}^{(r+1)}, \mathbf{X}^{(r+1)}, \mathbf{F}^{(r+1)}, \boldsymbol{\Theta}^{(r)}\right) \leq$ $\Phi\left(\mathbf{P}^{(r+1)}, \mathbf{X}^{(r+1)}, \mathbf{F}^{(r+1)}, \Theta^{(r+1)}\right)$.

As a result, based on the above analysis, we obtain

$$
\begin{aligned}
\Phi\left(\mathbf{P}^{(r)}, \mathbf{X}^{(r)}\right. & \left., \mathbf{F}^{(r)}, \Theta^{(r)}\right) \\
& \leq \Phi\left(\mathbf{P}^{(r+1)}, \mathbf{X}^{(r+1)}, \mathbf{F}^{(r+1)}, \Theta^{(r+1)}\right)
\end{aligned}
$$

which indicates that problem (20) is non-decreasing monotonically after each iteration. Since the objective function of problem (20) is upper bounded by a finite value, the proposed AOIA algorithm is guaranteed to converge.

Let us now discuss the computational complexity of the proposed AOIA algorithm. According to [36], [37, Lecture 6], convex problems involving linear matrix inequality (LMI), second-order cone (SOC) constraints and linear constraints that can be solved by a standard interior point method have a 
connotational complexity that can be formulated as follows

$$
\mathcal{O}\left(\left(\sum_{j=1}^{J} b_{j}+2 I\right)^{1 / 2} n(n^{2}+\underbrace{n \sum_{j=1}^{J} b_{j}^{2}+\sum_{j=1}^{J} b_{j}^{3}}_{\text {due to LMI }}+\underbrace{n \sum_{i=1}^{I} a_{i}^{2}}_{\text {due to SOC }})\right),
$$

where $n$ denotes the number of variables in the considered optimization problem, $J$ represents the number of LMIs (a linear constraint is equivalent to a LMI constraint) with dimension $b_{j}$, and $I$ is the number of SOC constraints with dimension $a_{i}$. More explicitly, in Step 3 of Algorithm 2, the complexity for solving problem (27) is $\mathfrak{o}_{\mathbf{P}, \mathbf{X}}=$ $\mathcal{O}\left(\sqrt{6} M L\left(M^{2} L^{2}+36 M L+216\right)\right)$. As for problem (27), there are $M$ LMIs with dimension $K$, thus in Step 4 of Algorithm 2, solving problem (31) results in a complexity of $\mathfrak{o}_{\mathbf{V}_{m}}=\mathcal{O}\left(M K^{1.5}\left(M^{2} K^{2}+M^{2} K^{3}+M K^{3}\right)\right)$. In Step 5, we obtain the optimal RIS reflection coefficients via Algorithm 1. Due to $N$ LMIs with 1 dimension, $L$ SOC constraints with $N$ dimensions and $N$ SOC constraints with 1 dimension, the complexity of solving problem (39) is $\mathfrak{o}_{\boldsymbol{\theta}}=$ $\mathcal{O}\left((2 L+3 N)^{1 / 2} n\left(n^{2}+(n+1) N+n\left(N^{2} L+N\right)\right)\right)$,

where $n=2 N+1$ is the number of variables. Accordingly, the computational complexity of Algorithm 1 is given by $i_{\max } \mathfrak{o}_{\boldsymbol{\theta}}$, where $i_{\max }$ is the number of iterations required for the penalty $\mathrm{CCP}$ algorithm to converge. Denoting by $r_{\max }$ the maximum number of iterations that allows Algorithm 2 to converge, the overall computational complexity of Algorithm 2 is thus equal to $r_{\max }\left(\mathfrak{o}_{\mathbf{P}, \mathbf{X}}+\mathfrak{o}_{\mathbf{V}_{m}}+i_{\max } \mathfrak{o}_{\boldsymbol{\theta}}\right)$.

Remark 3: Algorithm 2 solves the relaxed problem (20) where the binary spectrum sharing variables of the original problem in (7) are relaxed to continuous variables between 0 and 1. This relaxation is tight provided that the spectrum sharing variables $x_{m, l}$ are all binary, and the obtained solution is also a feasible solution of problem (7). Otherwise, the binary spectrum sharing solution needs to be reconstructed based on the solution obtained for problem (20). To this end, the reconstruction of $x_{m, l}$ is proceeded after the convergence of AOIA, and needs to maximize the objective function of problem (20). Since the $l$ th D2D-V2V pair reuses the spectrum pre-occupied by the $m$ th C-VUE, we select the optimal " $l *$ ". Hence, we have $l^{*}=\arg \max _{l}\left(\frac{\partial \sum_{m \in \mathcal{M}} R_{m}}{\partial x_{m, l}}\right)$, where $x_{m, l^{*}}=1$ indicates the optimal spectrum sharing variables for the $l$ th $\mathrm{D} 2 \mathrm{D}-\mathrm{V} 2 \mathrm{~V}$ pair.

\section{Simulation Results}

In this section, simulation results are presented to validate the proposed AOIA algorithm for application to resource sharing in RIS-aided vehicular networks. We customize the simulation setup for the freeway case detailed in 3GPP TR 36.885 [38], where a multi-lane highway is covered by a single cell with a radius of $500 \mathrm{~m}$ in which the BS is located at the center. Vehicles are dropped on the roads based on a spatial Poisson process and their velocity determines the vehicle density. The number of lanes, the lane width and the average distance between vehicles are the same as in [39].

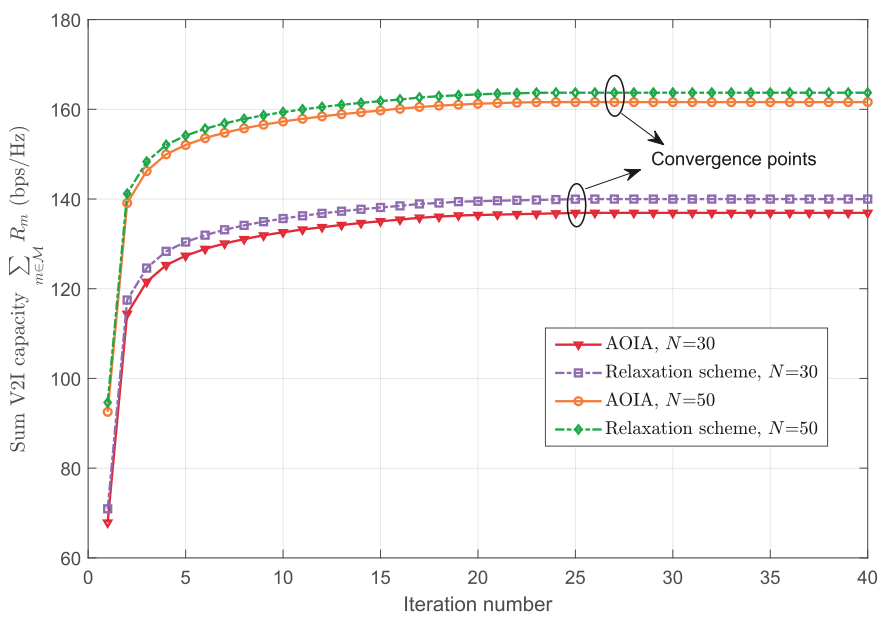

Fig. 2. Convergence behavior of Algorithm 2 parameterized by different $N$.

The three-dimensional coordinates of the BS and the RIS are $(0,0,25 \mathrm{~m})$ and $(100 \mathrm{~m}, 0,25 \mathrm{~m})$ respectively, which implies that the height of the RIS is assumed to be the same as that of the BS. The $M$ V2I links are randomly selected among the generated vehicles and the $L \mathrm{~V} 2 \mathrm{~V}$ links are formed between each vehicle with its closest surrounding neighbors, where all V2X links consist of direct links and RIS-aided reflection links. The path loss exponents of the BS-VUE, RIS-VUE and BS-RIS links are 3, 2.2 and 2.5, respectively. The Rician factors are all equal to $3 \mathrm{~dB}$. The BS is equipped with $K=32$ antennas and the total number of RIS reflecting elements is $N=N_{\mathrm{h}} \times N_{\mathrm{v}}(30=5 \times 6)$. The smooth parameter is $\delta=\frac{1}{\max _{\forall l \in \mathcal{L}}\left|R_{l}-R^{\mathrm{th}}\right|}$. As for Algorithm $1, \varpi=1.5$, $\lambda^{(0)}=0.1, \lambda_{\max }=300, \varepsilon^{\prime}=10^{-2}$, and $\varepsilon^{\prime \prime}=10^{-1}$. Other system parameters are set as follows unless specified otherwise: $\rho=-20 \mathrm{~dB}, P_{\max }^{c}=P_{\max }^{d}=23 \mathrm{dBm}$, $P_{\max }^{\text {outage }}=0.01, R^{\text {th }}=2.06 \mathrm{bps} / \mathrm{Hz}, M=L=10$, and $\sigma^{2}=-114 \mathrm{dBm}$.

Fig. 2 shows the convergence behavior of the proposed algorithm AOIA. The convergence condition is $\sum_{m \in \mathcal{M}}\left(R_{m}^{(r+1)}-R_{m}^{(r)}\right) / R_{m}^{(r+1)} \leq \epsilon(\epsilon=0.01)$ or $r \geq r_{\max }$. Since the initial values of the variables are randomly assigned at the beginning, the objective function is a relatively small value. Subsequently, the local optimal solution can be obtained step by step in each iteration, and then the algorithm gradually approaches a stationary solution. The objective function has a non-decreasing trend until it reaches the convergence condition, which is consistent with our analysis on the convergence behavior. In addition, a larger number of RIS reflection elements $N$ make the final objective function converge to a higher value.

For comparison, we consider the following five schemes as benchmarks. 1) Ideal scheme: we assume that the BS can access the instantaneous CSI of all V2X links; 2) Relaxation scheme: the binary spectrum variables $\mathbf{X}$ are not reconstructed, which thus provides an upper bound for AOIA; 3) Random $\Theta$ : the RIS reflection phase shift $\varphi_{n}$ randomly varies between 0 and $2 \pi$, i.e., removing the Step 5 in 


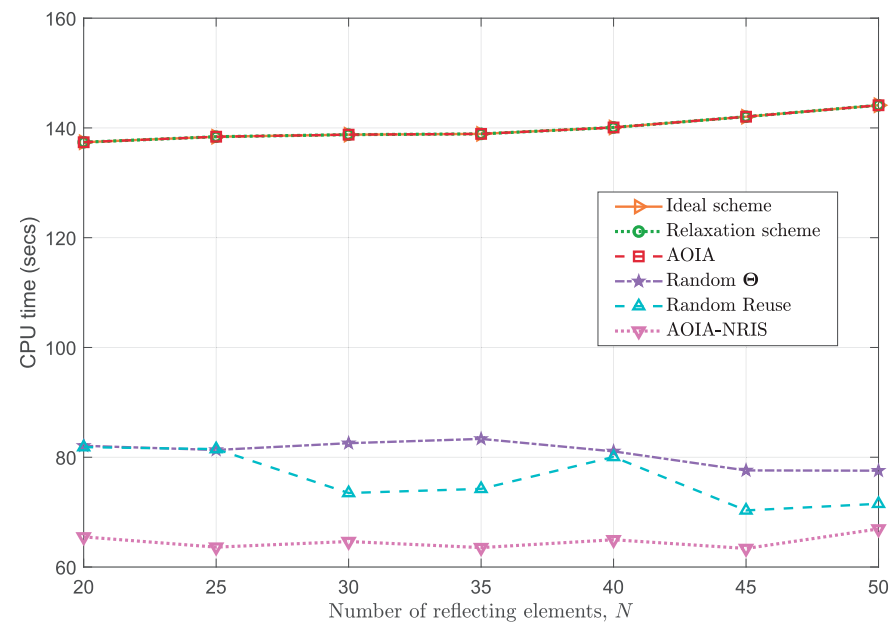

Fig. 3. Average CPU time versus the number of reflecting elements $N$.

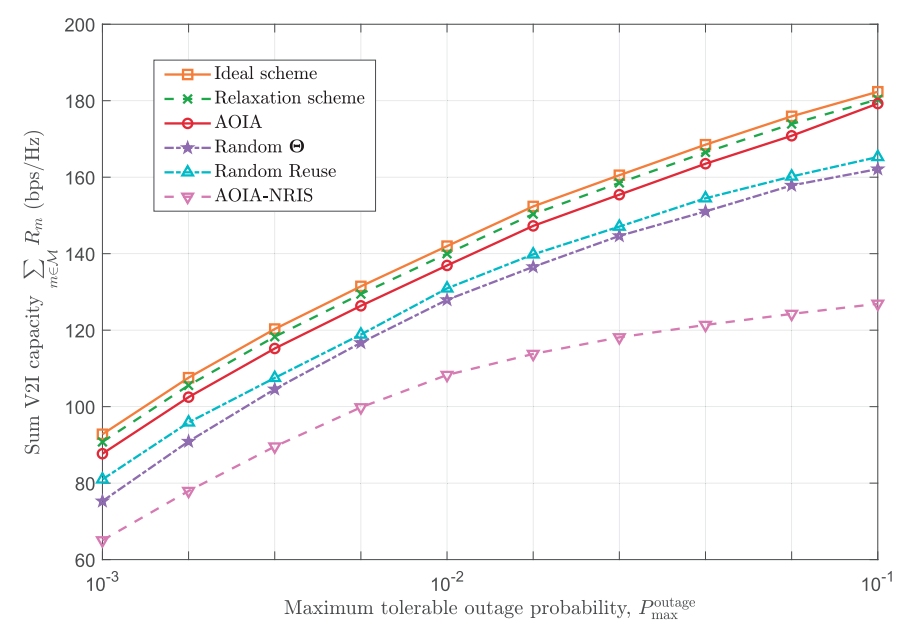

Fig. 4. Sum V2I capacity versus different maximum tolerable outage probability $P_{\max }^{\text {outage }}$.

Algorithm 2; 4) Random Reuse: the V2V links randomly reuse the spectrum preoccupied by the V2I links, i.e., the spectrum sharing variables $\mathbf{X}$ are not optimized in AOIA; 5) AOIA-NRIS: the considered vehicular network is not assisted by the RIS, which implies that the transmit power, the spectrum sharing and the MUD matrix are jointly optimized based on the proposed AOIA.

Fig. 3 compares the average central processing unit (CPU) running time of several proposed schemes versus the number of RIS reflecting elements $N$. The results are obtained by using a computer with a $1.61 \mathrm{GHz}$ i7-10710U CPU and $16 \mathrm{~GB}$ RAM. We observe that the CPU time increases as $N$ increases, as expected. Two random schemes spend relatively less CPU time than the ideal scheme, the relaxation scheme, and the AIOA scheme, due to the randomization of one of the blocks. In addition, our proposed AOIA algorithm provides theoretical guideline for resource allocation and beamforming design in RIS-aided vehicular networks. In practical scenarios, a more powerful arithmetic processor can provide a higher computing efficiency and thereby reduces the computational cost.

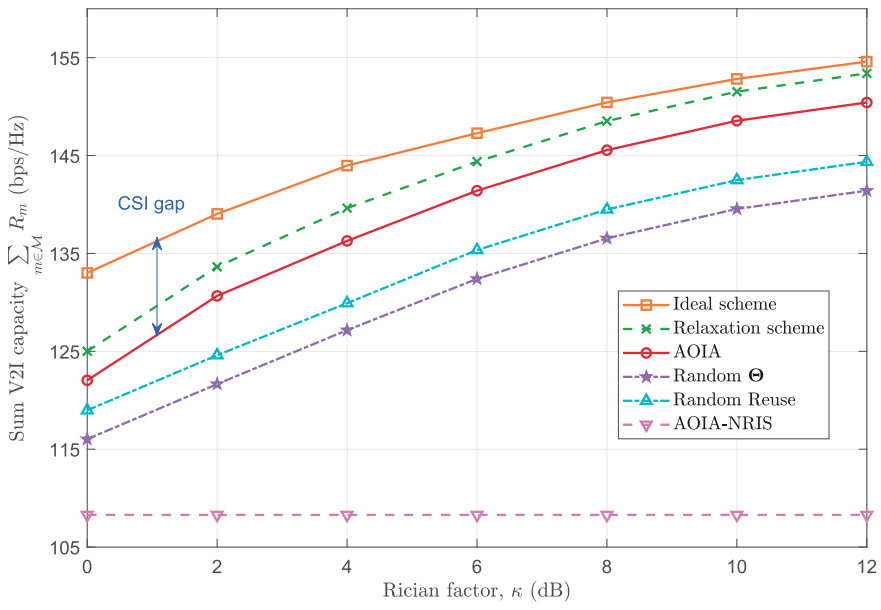

Fig. 5. Sum V2I capacity versus the Rician factor $\kappa$.

The impact of the maximum tolerable outage probability for V2V links on the sum V2I capacity is demonstrated in Fig. 4, which reveals that a higher maximum tolerable outage probability for the $\mathrm{V} 2 \mathrm{~V}$ links $P_{\max }^{\text {outage }}$ results in a larger sum V2I capacity. This is because a higher outage probability for the V2V links renders them more tolerant to the interference from the C-VUEs, which encourages the $\mathrm{C}$-VUEs to increase their transmit power. As a result, the sum V2I capacity increases. From another perspective, in light of the constraint (19), a higher $P_{\max }^{\text {outage }}$ allows (19) to have a smaller lower bound $\tilde{\gamma}^{\text {const }}$. Increasing $P_{m}^{c}$ appropriately caters to the change of the lower bound in (19), which leads to an increment in sum V2I capacity. Furthermore, the ideal scheme and relaxation scheme are always slightly superior to AOIA. This is due to a more accurate CSI that is exploited by the ideal scheme and an upper bound that is utilized in the relaxation scheme. In addition, as $P_{\max }^{\text {outage increases, compared }}$ to the scheme AOIA-NRIS, other RIS-aided schemes have a faster growth rate in terms of sum V2I capacity. Specifically, when $P_{\max }^{\text {outage }}$ varies from $10^{-2}$ to $10^{-1}$, the sum V2I capacity achieved by AOIA and AOIA-NRIS increases by $23.62 \%$ and $14.67 \%$, respectively. The $8.95 \%$ gain is due to the performance gain brought by RIS-aided V2X communications.

Fig. 5 plots the impact of different Rician factors on the sum V2I capacity. It is assumed that the BS-VUE link and the VUE-VUE link follow a Rayleigh distribution (no deterministic components exist due to blockage), i.e., $\kappa=0$, whilst both the BS-RIS link and the RIS-VUE link are assumed to follow a Rician distribution, i.e., $\kappa_{r, b}=\kappa_{m, r}=\kappa_{l, r}=\kappa_{r, l}=\kappa$. It is observed from Fig. 5 that the performance of the three schemes aided by RIS improves with $\kappa$. This phenomenon can be expected since both BS-RIS and RIS-VUE channels become more deterministic with an increment of $\kappa$, and a larger proportion of slowly varying LoS components can be obtained to boost the performance of the three RIS-aided schemes. Furthermore, the performance gap between the ideal scheme and AOIA eventually reduces, but cannot approach to zero. This phenomenon unmasks that CSI acquisition is a 


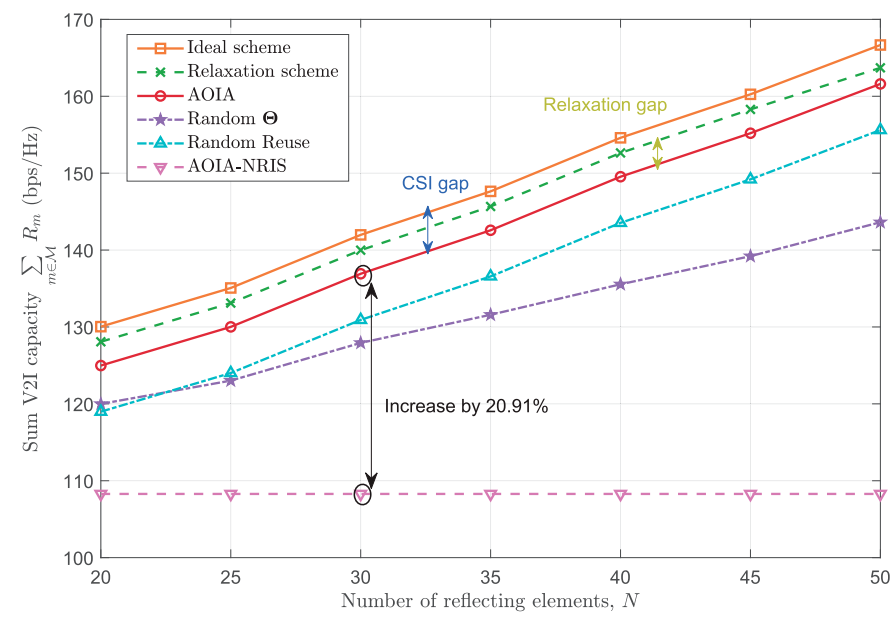

Fig. 6. Sum V2I capacity versus the number of reflecting elements $N$.

major bottleneck in the considered vehicular network, which limits the performance improvement. Additionally, the sum V2I capacity obtained by the AOIA-NRIS scheme is not sensitive to the change of the Rician factor. This is attributed to the fact that the corresponding direct links follow Rayleigh fading, and no LoS component can be obtained to further increase the sum V2I capacity.

In Fig. 6, we analyze the sum V2I capacity versus the number of RIS reflecting elements $N$ under different schemes. It is observed that the sum V2I capacity achieved by all RIS-aided schemes increases with $N$, while that of the AOIA-NRIS scheme remains unchanged. This is expected because more passive reflecting elements can reflect more signal power received from the BS, thereby resulting in greater power gain. To be specific, when $N=30$, compared with the AOIA-NRIS scheme, the sum V2I capacity obtained by AOIA increases by $20.91 \%$. In addition, the performance gain of the proposed AOIA algorithm becomes more pronounced as the number of reflecting elements increases and significantly outperforms other schemes, which highlights the importance of optimizing the RIS reflection coefficients optimization and the need for an elaborate design for spectrum sharing.

Fig. 7 shows the impact of RIS location on the sum V2I capacity. The BS and the RIS are located at $(0,0,25 \mathrm{~m})$ and $\left(x_{\mathrm{RIS}}, 0,25 \mathrm{~m}\right)$, respectively, and the distance between the BS and the RIS is denoted by $x_{\text {RIS }}(\mathrm{m})$. The path loss exponents of the BS-RIS link and the RIS-VUE link are all equal to 2.5. Since the vehicles are distributed according to a spatial Poisson process, we consider the distance between the BS and the VUE center point when studying the impact of the RIS location on the system performance. The distance between the BS and the VUE central point is set to $d_{\mathrm{VUE}}^{\mathrm{BS}}=160 \mathrm{~m}$. As illustrated in Fig. 7, the sum V2I capacity obtained by the RIS-aided schemes first decreases and then increases after achieving the minimum at $x_{\text {RIS }}=80 \mathrm{~m}$ when the RIS location is moved from $x_{\text {RIS }}=60 \mathrm{~m}$ to $x_{\text {RIS }}=100 \mathrm{~m}$. This performance trend can be explained as follows, by ignoring the small-scale fading for simplicity. The large-scale channel gain of the

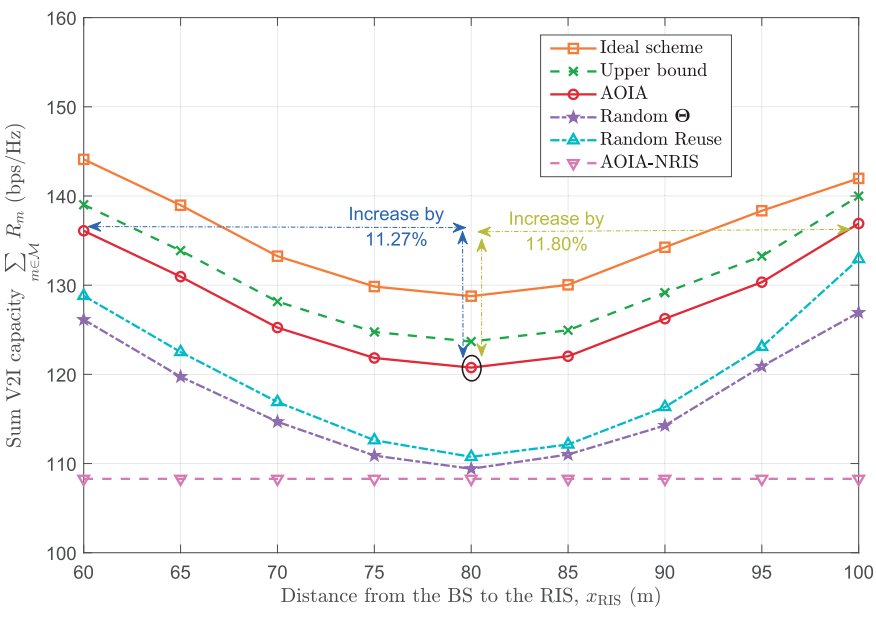

Fig. 7. Sum V2I capacity versus the location of the RIS coordinate $x_{\text {RIS }}$.

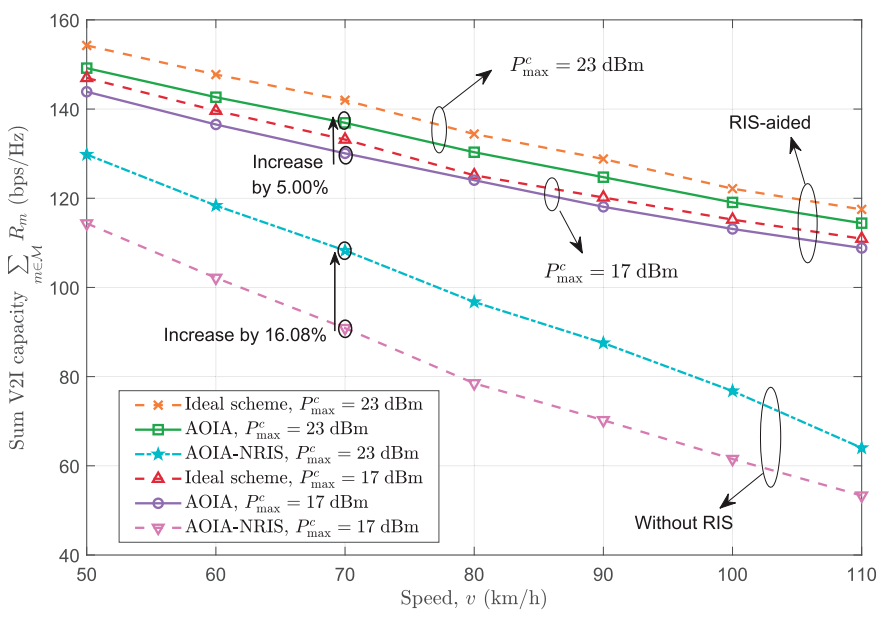

Fig. 8. Sum V2I capacity with varying vehicle speed $v$.

BS-RIS-VUE link can be approximated as

$$
\mathrm{PL}=10^{-2} \sqrt{\left(d_{\mathrm{RIS}}^{\mathrm{BS}} d_{\mathrm{VUE}}^{\mathrm{RIS}}\right)^{-2.5}}+\sqrt{10^{-2}\left(d_{\mathrm{VUE}}^{\mathrm{BS}}\right)^{-3}},
$$

where $d_{\mathrm{VUE}}^{\mathrm{BS}}=d_{\mathrm{RIS}}^{\mathrm{BS}}+d_{\mathrm{VUE}}^{\mathrm{RIS}}$. When $d_{\mathrm{RIS}}^{\mathrm{BS}}=d_{\mathrm{VUE}}^{\mathrm{RIS}}=\frac{1}{2} d_{\mathrm{VUE}}^{\mathrm{BS}}$, the combined channel gain achieves the minimum value, which explains the phenomenon in Fig. 7. Furthermore, when the vehicles are far away from the BS or they are far from each other, it does not mean that they have lower transmission rates. They may receive stronger reflected signals from the RIS. Compared with the location $x_{\text {RIS }}=80 \mathrm{~m}$, the sum V2I capacity increases by $11.27 \%$ and $11.80 \%$ at $x_{\text {RIS }}=$ $60 \mathrm{~m}$ and $x_{\text {RIS }}=100 \mathrm{~m}$, respectively. The results disclose that the system performance can be markedly enhanced by appropriately deploying the RIS.

In Fig. 8, we investigate the sum V2I capacity versus the vehicle speed $v$ by assuming $P_{\max }^{c}=P_{\max }^{d}$. It is observed that the sum V2I capacity achieved by all schemes decreases as $v$ increases, and the rate of decrease of the RIS-aided schemes is less than that of the schemes without RIS. Specifically, when the vehicle speed varies from $50 \mathrm{~km} / \mathrm{h}$ to $110 \mathrm{~km} / \mathrm{h}$ and $P_{\max }^{c}=P_{\max }^{d}=23 \mathrm{dBm}$, the sum V2I capacity 


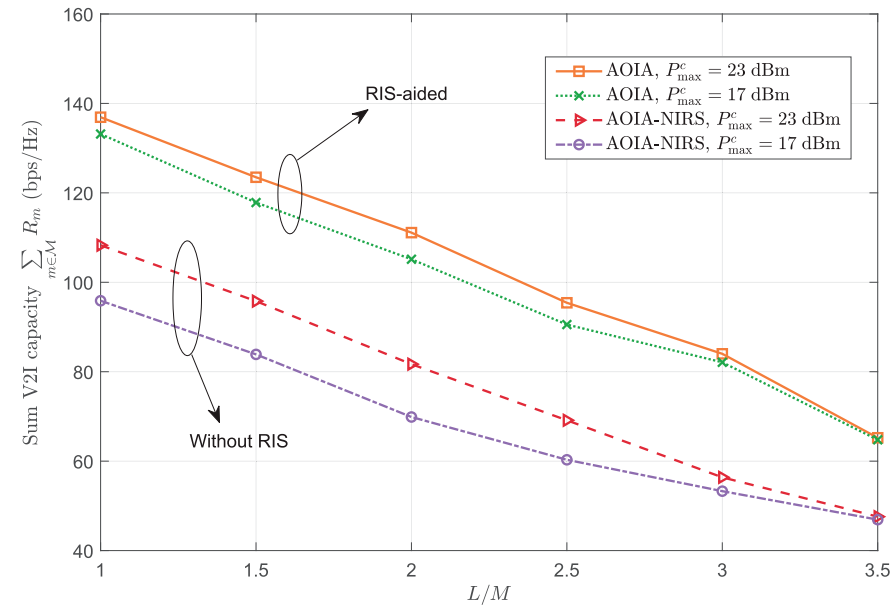

Fig. 9. Sum V2I capacity with varying number of V2V links.

obtained by AOIA decreases by $23.30 \%$, whereas that obtained by AOIA-NRIS decreases by $50.68 \%$. When the maximum transmit power increases by $6 \mathrm{dBm}$, in addition, the sum V2I capacity increases accordingly. In particular, the sum V2I capacity obtained by AOIA-NRIS increases by $16.08 \%$, while that obtained by AOIA increases by only $5.00 \%$. These phenomena can be attributed to the following facts. First, a higher speed induces sparser traffic according to the simulation setup, which, on average, increases inter-vehicle distance. To guarantee the reliability of the V2V links, the V2V transmitter needs to increase the transmit power to compensate for the higher path loss caused by the increased inter-vehicle distance, which in turns generates greater interference to the V2I links and thus limits the capacity of V2I links. Second, the reflected signal power compensates for part of the transmit power to achieve the same system capacity, which in turn reduces the transmit power. This explains why the performance gaps of RIS-aided schemes are smaller than those of the schemes without RIS.

To clarify the importance of resource sharing on the system performance for RIS-aided vehicular networks, the constraint $\sum_{m \in M} x_{m, l} \leq 1, \forall l$ in (7e) is removed in this simulation, which implies that the spectrum preoccupied by each V2I link can be shared by multiple V2V links. We set $M=10$ and $P_{\max }^{c}=P_{\max }^{d}$. From Fig. 9, it can be observed that the sum V2I capacity obtained by all schemes decreases as the number of $\mathrm{V} 2 \mathrm{~V}$ links increases. The main reasons for such capacity degradation are as follows. Each V2I link needs to share spectrum with more active $\mathrm{V} 2 \mathrm{~V}$ links simultaneously. To ensure the reliability of these $\mathrm{V} 2 \mathrm{~V}$ links, the transmit power of the C-VUE needs to be controlled to reduce the interference to $\mathrm{V} 2 \mathrm{~V}$ links. The decreased signal power of each V2I link causes the degradation of the sum V2I capacity. Furthermore, an increasing number of $\mathrm{V} 2 \mathrm{~V}$ links give rise to more interference to the V2I links, which reduces $\mathrm{SINR}_{m}$. In addition, when the maximum transmit power is increased from $17 \mathrm{dBm}$ to $23 \mathrm{dBm}$, the sum V2I capacity increases accordingly, and the performance gaps of the RIS-aided schemes are smaller than those of the schemes without RIS. When $\frac{L}{M} \geq 3$, however, the capacity gain is marginal, and the gap between the two is close. It can be concluded that the V2I links suffer severe interference from the V2V links under these circumstances, which constitutes the dominant factor that negatively affects the sum V2I capacity.

\section{CONCLUSION}

In this work, to enhance the ubiquitous coverage and QoS guarantee of V2X communications, we demonstrate the performance benefits of deploying RISs in vehicular networks. Based on a spectrum sharing model, a sum-V2I-capacity-maximization problem is formulated, subject to practical QoS requirements and RIS phases shifts. In particular, an effective algorithm based on alternating optimization is developed in order to jointly optimize the transmit power, the MUD matrix, the spectrum sharing of the V2V links, and the RIS reflection coefficients. The simulation results demonstrate the significant enhancement of the sum V2I capacity provided by our proposed algorithm while guaranteeing the reliability of the $\mathrm{V} 2 \mathrm{~V}$ links under various system configurations. In addition, it is shown that the use of an RIS can compensate for the system performance loss due to the high movement of vehicles and significant gains can be obtained by carefully choosing the location of the RIS, which provides valuable guidance for developing new paradigms for future vehicular networks.

\section{APPENDIX I \\ PROOF OF THEOREM 1}

The core idea for proving Theorem $l$ is to check the Karush-Kuhn-Tucker (KKT) optimality conditions of problem (31). For simplicity, we introduce auxiliary variables $\boldsymbol{\tau}=\left\{\tau_{m}=P_{m}^{c} \operatorname{Tr}\left(\mathbf{H}_{m} \mathbf{V}_{m}\right)+\sum_{l \in \mathcal{L}} x_{m, l} P_{l}^{d} \operatorname{Tr}\left(\mathbf{H}_{l} \mathbf{V}_{m}\right)\right.$ $\left.+\sigma^{2} \operatorname{Tr}\left(\mathbf{V}_{m}\right), \forall m\right\}$. Thus, problem (31) can be transformed into an equivalent problem

$$
\begin{aligned}
\max _{\left\{\mathbf{V}_{m}, \boldsymbol{\tau}\right\}} & \sum_{m \in \mathcal{M}}\left(\log _{2} \tau_{m}-\tilde{R}_{m}^{u b}\right) \\
\text { s.t. } & \mathbf{V}_{m} \succeq 0, \forall m, \\
& P_{m}^{c} \operatorname{Tr}\left(\mathbf{H}_{m} \mathbf{V}_{m}\right)+\sum_{l \in \mathcal{L}} x_{m, l} P_{l}^{d} \operatorname{Tr}\left(\mathbf{H}_{l} \mathbf{V}_{m}\right) \\
& +\sigma^{2} \operatorname{Tr}\left(\mathbf{V}_{m}\right) \geq \tau_{m}, \forall m .
\end{aligned}
$$

Problem (46) is convex and satisfies Slater's condition, so the strong duality holds [32]. Let $\mathbf{D}_{m} \in \mathbb{C}^{K \times K}$ and $\iota_{m}$ denote the dual variables associated with constraints (46b) and (46c), respectively. The Lagrangian function of problem (46) can be written as

$$
\begin{aligned}
\mathfrak{L}\left(\mathbf{V}_{m}, \tau_{m}, \iota_{m}, \mathbf{D}_{m}\right) \triangleq & \sum_{m \in \mathcal{M}}\left(\log _{2} \tau_{m}-\tilde{R}_{m}^{u b}\right) \\
& +\sum_{m \in \mathcal{M}} \operatorname{Tr}\left(\mathbf{D}_{m} \mathbf{V}_{m}\right)
\end{aligned}
$$




$$
\mathbf{u}^{H} \mathbf{D}_{m}^{*} \mathbf{u}=\mathbf{u}^{H}\left[\left(\Omega_{m}^{-1}-\iota_{m}^{*}\right) \sigma^{2} \mathbf{I}_{K}+\Omega_{m}^{-1} \sum_{l \in \mathcal{L}} x_{m, l} P_{l}^{d} \mathbf{H}_{l}-\iota_{m}^{*}\left(P_{m}^{c} \mathbf{H}_{m}+\sum_{l \in \mathcal{L}} x_{m, l} P_{l}^{d} \mathbf{H}_{l}\right)\right] \mathbf{u} \geq 0
$$

$$
\begin{aligned}
& +\iota_{m}\left[P_{m}^{c} \operatorname{Tr}\left(\mathbf{H}_{m} \mathbf{V}_{m}\right)+\sum_{l \in \mathcal{L}} x_{m, l} P_{l}^{d}\right. \\
& \left.\operatorname{Tr}\left(\mathbf{H}_{l} \mathbf{V}_{m}\right)+\sigma^{2} \operatorname{Tr}\left(\mathbf{V}_{m}\right)-\tau_{m}\right]
\end{aligned}
$$

The dual problem of (46) is given by

$$
\max _{\mathbf{D}_{m} 0, \iota_{m} \geq 0} \min _{\mathbf{V}_{m}, \tau_{m}} \mathfrak{L}\left(\mathbf{V}_{m}, \tau_{m}, \iota_{m}, \mathbf{D}_{m}\right) .
$$

Then we investigate the structure of the optimal solution $\mathbf{V}_{m}^{*}$ of the dual problem (48) according to the KKT conditions. Specifically, the KKT conditions associated with $\mathbf{V}_{m}^{*}$ are given by

$$
\tau_{m}^{*} \geq 0, \iota_{m}^{*} \geq 0 ; \mathbf{D}_{m}^{*} \succeq 0 ; \mathbf{D}_{m}^{*} \mathbf{V}_{m}^{*}=0 ; \quad \nabla_{\mathbf{V}_{m}^{*}} \mathfrak{L}=0 .
$$

In (49), $\tau_{m}^{*}, \iota_{m}^{*}$ and $\mathbf{D}_{m}^{*}$ are the optimal dual variables of problem (48). To facilitate the proof, the first-order optimal condition $\nabla_{\mathbf{V}_{m}^{*}} \mathfrak{L}=0$ with respect to $\mathbf{V}_{m}^{*}$ can be explicitly rewritten as $\mathbf{D}_{m}^{*}=\left(\Omega_{m}^{-1}-\iota_{m}^{*}\right) \sigma^{2} \mathbf{I}_{K}+\boldsymbol{\Xi}_{m}$, where $\boldsymbol{\Xi}_{m}=\Omega_{m}^{-1} \sum_{l \in \mathcal{L}} x_{m, l} P_{l}^{d} \mathbf{H}_{l}-\iota_{m}^{*}\left(P_{m}^{c} \mathbf{H}_{m}+\sum_{l \in \mathcal{L}} x_{m, l} P_{l}^{d} \mathbf{H}_{l}\right)$. It is observed that the value of the dual variable $\iota_{m}^{*}$ affects the structure of $\mathbf{D}_{m}^{*}$. Next, we show that regardless of the value taken by $\iota_{m}^{*}$ in the domain, $\operatorname{Rank}\left(\mathbf{D}_{m}^{*}\right) \geq K-1$ always holds.

Define a set $\Psi \triangleq\left\{m \mid \iota_{m}^{*} \geq 0, \iota_{m}^{*}=\Omega_{m}^{-1}, \forall m \in \mathcal{M}\right\}$, where $\Psi \neq \emptyset$. $\mathbf{D}_{m}^{*}$ can be expressed as

$$
\mathbf{D}_{m}^{*}=\left\{\begin{array}{cc}
\boldsymbol{\Xi}_{m}, & \text { if } m \in \Psi, \\
\left(\Omega_{m}^{-1}-\iota_{m}^{*}\right) \sigma^{2} \mathbf{I}_{K}+\boldsymbol{\Xi}_{m}, & \text { otherwise. }
\end{array}\right.
$$

Since $\mathbf{D}_{m}^{*} \succeq 0$ and $-\iota_{m}^{*}\left(P_{m}^{c} \mathbf{H}_{m}+\sum_{l \in \mathcal{L}} x_{m, l} P_{l}^{d} \mathbf{H}_{l}\right) \preceq 0$, it alaways follows that $\boldsymbol{\Xi}_{m} \succeq 0$ regardless of the value that $\iota_{m}^{*}$ takes in the domain. In the following, we will discuss the rank of $\mathbf{D}_{m}^{*}$ for $m \in \Psi$ and $m \notin \Psi$.

1) If $m \notin \Psi$, we show that $\left(\Omega_{m}^{-1}-\iota_{m}^{*}\right) \sigma^{2} \mathbf{I}_{K}+$ $\Omega_{m}^{-1} \sum_{l \in \mathcal{L}} x_{m, l} P_{l}^{d} \mathbf{H}_{l}$ is full rank by contradiction. If it is not full-rank, suppose that there exits a non-zero vector $\mathbf{u} \neq 0$ that satisfies $\mathbf{u}^{H}\left(\left(\Omega_{m}^{-1}-\iota_{m}^{*}\right) \sigma^{2} \mathbf{I}_{K}+\Omega_{m}^{-1} \sum_{l \in \mathcal{L}} x_{m, l} P_{l}^{d} \mathbf{H}_{l}\right) \mathbf{u}=0$. Since $\mathbf{D}_{m}^{*} \succeq 0$, we have (51), shown at the top of the page, which implies $-\iota_{m}^{*}\left(P_{m}^{c} \mathbf{u}^{H} \mathbf{H}_{m} \mathbf{u}+\sum_{l \in \mathcal{L}} x_{m, l} P_{l}^{d} \mathbf{u}^{H} \mathbf{H}_{l} \mathbf{u}\right) \geq 0$. Therefore, it holds that $\mathbf{u}^{H} \mathbf{H}_{m} \mathbf{u}=0$ and $\mathbf{u}^{H} \mathbf{H}_{l} \mathbf{u}=0$. As for $\left(\Omega_{m}^{-1}-\iota_{m}^{*}\right) \sigma^{2} \mathbf{I}_{K}+\Omega_{m}^{-1} \sum_{l \in \mathcal{L}} x_{m, l} P_{l}^{d} \mathbf{H}_{l}$, we have

$$
\begin{aligned}
\mathbf{u}^{H}\left(\left(\Omega_{m}^{-1}-\iota_{m}^{*}\right)\right. & \left.\sigma^{2} \mathbf{I}_{K}+\Omega_{m}^{-1} \sum_{l \in \mathcal{L}} x_{m, l} P_{l}^{d} \mathbf{H}_{l}\right) \mathbf{u} \\
& =\left(\Omega_{m}^{-1}-\iota_{m}^{*}\right) \sigma^{2} \mathbf{u}^{H} \mathbf{u} \neq 0, m \notin \Psi,
\end{aligned}
$$

which contradicts the assumption. Thus it must hold that $\left(\Omega_{m}^{-1}-\iota_{m}^{*}\right) \sigma^{2} \mathbf{I}_{K}+\Omega_{m}^{-1} \sum_{l \in \mathcal{L}} x_{m, l} P_{l}^{d} \mathbf{H}_{l} \succ 0$, which implies that this matrix must be full rank. As a result, if $m \notin \Psi$, the rank of $\mathbf{D}_{m}^{*}$ is at least $K-1$.

2) If $m \in \Psi$, we have $\mathbf{D}_{m}^{*}=\boldsymbol{\Xi}_{m}$. It is not difficult to show that matrix $\Omega_{m}^{-1} \sum_{l \in \mathcal{L}} x_{m, l} P_{l}^{d} \mathbf{H}_{l}$ is full rank by using steps similar to the previous case study. Thus $\operatorname{Rank}\left(\mathbf{D}_{m}^{*}\right) \geq K-1$ holds when $m \in \Psi$, we have $\mathbf{D}_{m}^{*}=\boldsymbol{\Xi}_{m}$.

Based on the proofs of both parts, it always holds that $\operatorname{Rank}\left(\mathbf{D}_{m}^{*}\right) \geq K-1$. Combining the KKT conditions $\mathbf{D}_{m}^{*} \mathbf{V}_{m}^{*}=0$, we have $\operatorname{Rank}\left(\mathbf{V}_{m}^{*}\right)=1$ and the proof of Theorem 1 is thus completed.

\section{REFERENCES}

[1] R. Molina-Masegosa and J. Gozalvez, "LTE-V for sidelink 5G V2X vehicular communications: A new 5G technology for shortrange vehicle-to-everything communications," IEEE Veh. Technol. Mag., vol. 12, no. 4, pp. 30-39, Dec. 2017.

[2] Technical Specification Group Radio Access Network; Study Enhancement 3GPP Support for 5G V2X Services; (Release 15), document TR 22.886 V15.1.0, 3GPP, Mar. 2017.

[3] Technical Specification Group Services and System Aspects; Enhancement of 3GPP Support for V2X Scenarios; (Release 16), document TS 22.186 V16.2.0, V14.0.0, 3GPP, Jun. 2019.

[4] S. Zeadally, M. A. Javed, and E. B. Hamida, "Vehicular communications for ITS: Standardization and challenges," IEEE Commun. Standards Mag., vol. 4, no. 1, pp. 11-17, Mar. 2020.

[5] J. Zhang, E. Björnson, M. Matthaiou, D. W. K. Ng, H. Yang, and D. J. Love, "Prospective multiple antenna technologies for beyond 5G," IEEE J. Sel. Areas Commun., vol. 38, no. 8, pp. 1637-1660, Aug. 2020.

[6] M. Di Renzo et al., "Smart radio environments empowered by reconfigurable intelligent surfaces: How it works, state of research, and the road ahead," IEEE J. Sel. Areas Commun., vol. 38, no. 11, pp. 2450-2525, Nov. 2020.

[7] C. Huang et al., "Holographic MIMO surfaces for $6 \mathrm{G}$ wireless networks: Opportunities, challenges, and trends," IEEE Wireless Commun., vol. 27, no. 5, pp. 118-125, Oct. 2020.

[8] S. Abeywickrama, R. Zhang, Q. Wu, and C. Yuen, "Intelligent reflecting surface: Practical phase shift model and beamforming optimization," IEEE Trans. Commun., vol. 68, no. 9, pp. 5849-5863, Sep. 2020.

[9] Q. Wu and R. Zhang, "Beamforming optimization for wireless network aided by intelligent reflecting surface with discrete phase shifts," IEEE Trans. Commun., vol. 68, no. 3, pp. 1838-1851, Mar. 2020.

[10] X. Yu, D. Xu, Y. Sun, D. W. K. Ng, and R. Schober, "Robust and secure wireless communications via intelligent reflecting surfaces," IEEE J. Sel. Areas Commun., vol. 38, no. 11, pp. 2637-2652, Nov. 2020.

[11] M. Di Renzo et al., "Smart radio environments empowered by reconfigurable AI meta-surfaces: An idea whose time has come," EURASIP J. Wireless Commun. Netw., vol. 2019, no. 1, pp. 1-20, May 2019.

[12] S. Li, B. Duo, X. Yuan, Y.-C. Liang, and M. Di Renzo, "Reconfigurable intelligent surface assisted UAV communication: Joint trajectory design and passive beamforming," IEEE Wireless Commun. Lett., vol. 9, no. 5, pp. 716-720, May 2020.

[13] G. Zhou, C. Pan, H. Ren, K. Wang, and A. Nallanathan, "Intelligent reflecting surface aided multigroup multicast MISO communication systems," IEEE Trans. Signal Process., vol. 68, pp. 3236-3251, Apr. 2020.

[14] C. Pan et al., "Multicell MIMO communications relying on intelligent reflecting surfaces," IEEE Trans. Wireless Commun., vol. 19, no. 8, pp. 5218-5233, Aug. 2020.

[15] T. Bai, C. Pan, Y. Deng, M. Elkashlan, A. Nallanathan, and L. Hanzo, "Latency minimization for intelligent reflecting surface aided mobile edge computing," IEEE J. Sel. Areas Commun., vol. 38, no. 11, pp. 2666-2682, Nov. 2020. 
[16] C. Huang, A. Zappone, G. C. Alexandropoulos, M. Debbah, and C. Yuen, "Reconfigurable intelligent surfaces for energy efficiency in wireless communication," IEEE Trans. Wireless Commun., vol. 18, no. 8, pp. 4157-4170, Aug. 2019.

[17] Y. Yang, S. Zhang, and R. Zhang, "IRS-enhanced OFDMA: Joint resource allocation and passive beamforming optimization," IEEE Wireless Commun. Lett., vol. 9, no. 6, pp. 760-764, Jun. 2020.

[18] D. Xu, X. Yu, Y. Sun, D. W. K. Ng, and R. Schober, "Resource allocation for secure IRS-assisted multiuser MISO systems," in Proc. IEEE Globecom Workshops (GC Wkshps), Waikoloa, HI, USA, Dec. 2019, pp. 1-6.

[19] Q. Wu and R. Zhang, "Joint active and passive beamforming optimization for intelligent reflecting surface assisted SWIPT under QoS constraints," IEEE J. Sel. Areas Commun., vol. 38, no. 8, pp. 1735-1748, Aug. 2020.

[20] A. U. Makarfi et al., "Reconfigurable intelligent surfaces-enabled vehicular networks: A physical layer security perspective," 2020 , arXiv:2004.11288. [Online]. Available: http://arxiv.org/abs/2004.11288

[21] J. Wang, W. Zhang, X. Bao, T. Song, and C. Pan, "Outage analysis for intelligent reflecting surface assisted vehicular communication networks," in Proc. IEEE Global Commun. Conf. (GLOBECOM), Taipei, Taiwan, Dec. 2020, pp. 1-6.

[22] L. Liang, H. Ye, and G. Y. Li, "Spectrum sharing in vehicular networks based on multi-agent reinforcement learning," IEEE J. Sel. Areas Commun., vol. 37, no. 10, pp. 2282-2292, Oct. 2019.

[23] C. Guo, L. Liang, and G. Y. Li, "Resource allocation for vehicular communications with low latency and high reliability," IEEE Trans. Wireless Commun., vol. 18, no. 8, pp. 3887-3902, Aug. 2019.

[24] Y. Chen, Y. Wang, J. Zhang, and Z. Li, "Resource allocation for intelligent reflecting surface aided vehicular communications," IEEE Trans. Veh. Technol., vol. 69, no. 10, pp. 12321-12326, Oct. 2020.

[25] H. Yang, K. Zhang, K. Zheng, and Y. Qian, "Joint frame design and resource allocation for ultra-reliable and low-latency vehicular networks," IEEE Trans. Wireless Commun., vol. 19, no. 5, pp. 3607-3622, May 2020.

[26] C. Guo, L. Liang, and G. Y. Li, "Resource allocation for low-latency vehicular communications: An effective capacity perspective," IEEE J. Sel. Areas Commun., vol. 37, no. 4, pp. 905-917, Apr. 2019.

[27] L. Liang, S. Xie, G. Y. Li, Z. Ding, and X. Yu, "Graph-based resource sharing in vehicular communication," IEEE Trans. Wireless Commun., vol. 17, no. 7, pp. 4579-4592, Jul. 2018.

[28] A. Liu, V. K. N. Lau, and B. Kananian, "Stochastic successive convex approximation for non-convex constrained stochastic optimization," IEEE Trans. Signal Process., vol. 67, no. 16, pp. 4189-4203, Aug. 2019.

[29] Q. Zhang, S. Jin, K.-K. Wong, H. Zhu, and M. Matthaiou, "Power scaling of uplink massive MIMO systems with arbitrary-rank channel means," IEEE J. Sel. Topics Signal Process., vol. 8, no. 5, pp. 966-981, Oct. 2014.

[30] M. K. Simon, Probability Distributions Involving Gaussian Random Variables: A Handbook for Engineers and Scientists. Springer, 2007.

[31] C.-Y. Chi, W.-C. Li, and C.-H. Lin, Convex Optimization for Signal Processing and Communications: From Fundamentals to Applications. Boca Raton, FL, USA: CRC Press, 2017.

[32] S. Boyd and L. Vandenberghe, Convex Optimization. Cambridge, U.K.: Cambridge Univ. Press, 2004.

[33] M. Grant and S. Boyd. (Mar. 2014). CVX: MATLAB Software for Disciplined Convex Programming, Version 2.1. [Online]. Available: http://cvxr.com/cvx

[34] Mosek Optimization Toolbox for MATLAB, Release 9.2.29, User's Guide Reference Manual, MOSEK ApS, Copenhagen, Denmark, Oct. 2020, vol. 4.

[35] T. Lipp and S. Boyd, "Variations and extension of the convex-concave procedure," Optim. Eng., vol. 17, no. 2, pp. 263-287, Jun. 2016.

[36] A. Ben-Tal and A. Nemirovski, Lectures on Modern Convex Optimization: Analysis, Algorithms, and Engineering Applications. Philadelphia, PA, USA: SIAM, 2001

[37] K.-Y. Wang, A. M.-C. So, T.-H. Chang, W.-K. Ma, and C.-Y. Chi, "Outage constrained robust transmit optimization for multiuser MISO downlinks: Tractable approximations by conic optimization," IEEE Trans. Signal Process., vol. 62, no. 21, pp. 5690-5705, Nov. 2014.

[38] Technical Specification Group Radio Access Network; Study LTE-Based V2X Services; (Release 14), document TR 36.885 V14.0.0, 3GPP, Jun. 2016
[39] Y. Chen, Y. Wang, M. Liu, J. Zhang, and L. Jiao, "Network slicing enabled resource management for service-oriented ultra-reliable and low-latency vehicular networks," IEEE Trans. Veh. Technol., vol. 69, no. 7, pp. 7847-7862, Jul. 2020. 